\title{
Review Article \\ Hydroxysafflor Yellow A: A Promising Therapeutic Agent for a Broad Spectrum of Diseases
}

\author{
Hui Ao $\mathbb{D},^{1,2}$ Wuwen Feng $\mathbb{D},{ }^{1}$ and Cheng Peng $\mathbb{D}^{1}$ \\ ${ }^{1}$ College of Pharmacy, Chengdu University of Traditional Chinese Medicine, Chengdu 611137, China \\ ${ }^{2}$ Innovative Institute of Chinese Medicine and Pharmacy, Chengdu University of Traditional Chinese Medicine, Chengdu 611137, China \\ Correspondence should be addressed to Cheng Peng; pengchengcxy@126.com
}

Received 9 May 2018; Accepted 12 August 2018; Published 25 September 2018

Academic Editor: Ching-Liang Hsieh

Copyright (c) 2018 Hui Ao et al. This is an open access article distributed under the Creative Commons Attribution License, which permits unrestricted use, distribution, and reproduction in any medium, provided the original work is properly cited.

\begin{abstract}
Hydroxysafflor yellow A (HSYA) is one of the major bioactive and water-soluble compounds isolated from Carthami Flos, the flower of safflower (Carthamus tinctorius L.). As a natural pigment with favorable medical use, HSYA has gained extensive attention due to broad and effective pharmacological activities since first isolation in 1993. In clinic, the safflor yellow injection which mainly contains about $80 \%$ HSYA was approved by the China State Food and Drug Administration and used to treat cardiac diseases such as angina pectoris. In basic pharmacology, HSYA has been proved to exhibit a broad spectrum of biological effects that include, but not limited to, cardiovascular effect, neuroprotection, liver and lung protection, antitumor activity, metabolism regulation, and endothelium cell protection. Although a great number of studies have been carried out to prove the pharmacological effects and corresponding mechanisms of HYSA, a systemic review of HYSA has not yet been seen. Here, we provide a comprehensive summarization of the pharmacological effects of HYSA. Together with special attention to mechanisms of actions, this review can serve as the basis for further researches and developments of this medicinal compound.
\end{abstract}

\section{Instruction}

Carthamus tinctorius L. (Figure 1(a)), also named safflower, belongs to the genus Carthamus family Compositae. As a multipurpose cash crop in agriculture, industry, and medicine, it is cultivated for its seeds, meals, and flowers. In terms of medical use, safflower is widely applied in East Asia especially in China [1].

Carthami Flos (Figure 1(b)), also named Honghua in China, is the dried floret of safflower and known as a blood stasis promoting herb. Golden Chamber Synopsis (Jin Gui Yao Nüe) by Zhang Zhongjing in the Han Danasty deemed the decoction of Carthami Flos as an effective remedy for gynaecological problems. Carthami Flos was firstly introduced as a medicinal herb in Annotation of Materia Medica (Xin Xiu Ben Cao) of the Tang Dynasty for the treatment of lockjaw, hemonode, and postpartum illness. Since then, the extracts of Carthami Flos have been extensively applied to treat several diseases such as cardiovascular and cerebrovascular disorders caused by blood stasis. (a) Carthamus tinctorius L. (Safflower). (b) Carthami Flos (the dried flower of Carthamus tinctorius L.). (c) Hydroxysafflor yellow A (the main effective compound of Carthami Flos). Hydroxysafflor yellow A (HYSA, Figure 1(c)) is a watersoluble compound mainly responsible for the medicinal activities of Carthami Flos. It was firstly separated from Carthamus tinctorius L. by Meselhy et al. in 1993 [2] and is regarded as one of the standard components for quality control of Carthami Flos according to the Chinese Pharmacopoeia, because of its rich abundance and strong activities [3]. In 2005, the Safflor yellow injection which contained 45 mg HSYA per $50 \mathrm{mg}$ was approved as a novel cardiovascular drug by China State Food and Drug Administration and began to be widely used for treatment of cardiac diseases such as angina pectoris. The systematic evaluations demonstrated that this injection was significantly effective for both angina pectoris and cerebral infarction $[4,5]$. Apart from the effect on cardiovascular disorders, HYSA showed neuroprotection, anticancer properties, and metabolism regulation as well as liver, lung, and endothelium cell protection. In this review, 


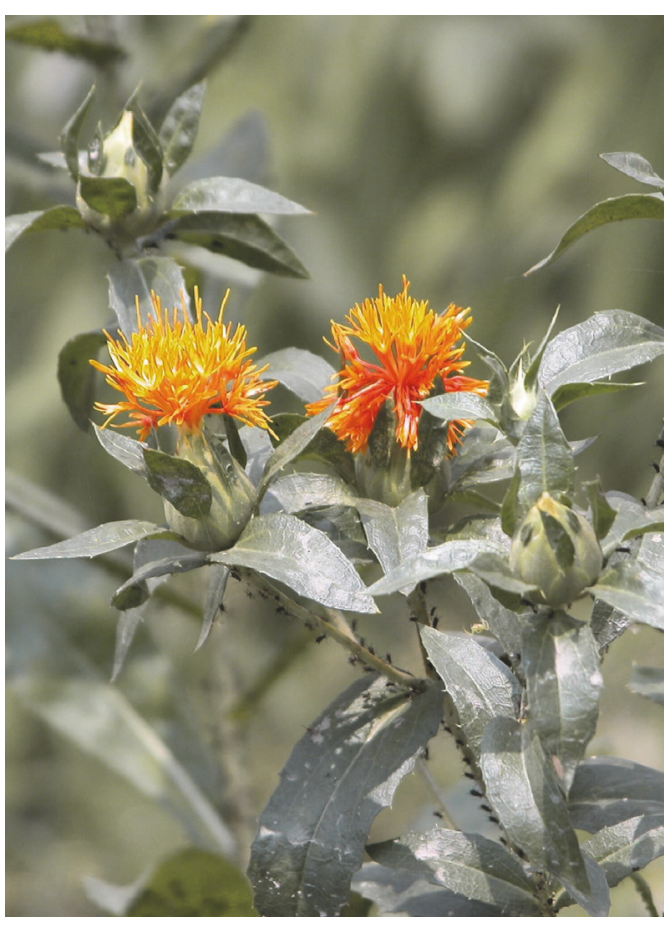

(a)

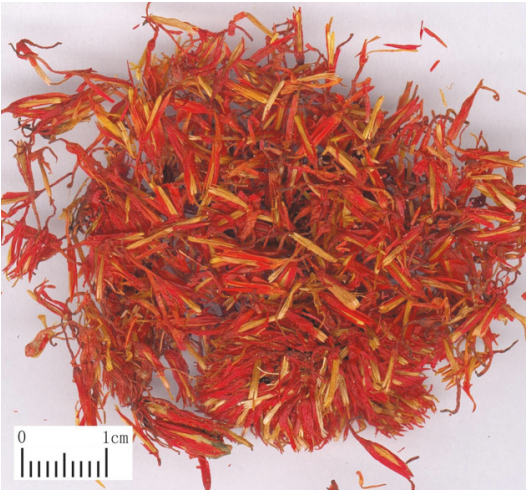

(b)<smiles>O=C(/C=C/c1ccc(O)cc1)C1=C(O)C2(O)C(O)=C(C3OC(CO)C(O)C(O)C3O)C(=O)C(O)C2OC(CO)C1O</smiles>

(c)

FIgURE 1: Hydroxysafflor yellow A (HYSA) and its sources.

we managed to give a comprehensive review and analysis of the pharmacological properties of HYSA, supporting the potential application of HYSA in clinic.

\section{Pharmacology}

2.1. Cardiovascular Effects. In clinic, products containing HYSA are mostly used for treatment of cardiovascular diseases. The therapeutic effect of HYSA on cardiovascular diseases is related to its anticoagulant effect, antimyocardial ischemia activity, vasorelaxative effect, etc.

2.1.1. Anticoagulant Effect. The anticoagulant action of HYSA was investigated mainly in vitro. HYSA could elicit suppressive effects on thrombosis formation in the normal rats and rabbit platelet aggregation induced by adenosine diphosphate (ADP) and platelet activating factor (PAF) as well as rabbit blood viscosity ex vivo $[6,7]$. It could also prolong prothrombin time (PT) of rat plasma and recalcification time (RT) of rabbit plasma in vitro [8].

2.1.2. Effect on Myocardial Ischemia. As an antiangina drug, the cardiac protection of HYSA has been observed in vivo and in vitro. HSYA could reverse the haemodynamic alteration, enhance the survival rate, alleviate the myocardial damage, and promote the angiogenesis in the ischemic myocardium by enhancing expression of nucleolin and thus upregulating expressions of vascular endothelial growth factor $\mathrm{A}$ (VEGF-A) and matrix metallopeptidase 9 (MMP-9) in the myocardial ischemia (MI) rats induced by occlusion of left anterior descending coronary artery (LAD) [9]. In addition, HYSA could also lower the levels of cardiac troponin I (cTnI) and 8-hydroxy-2'-deoxyguanosine (8-OHdG) in the MI mice induced by LAD [10]. In vitro, HYSA could relieve the nuclear morphological changes and levels of 
malondialdehyde (MDA) and reactive oxygen species (ROS), lower the activities of creatine kinase-MB (CK-MB) and lactate dehydrogenase (LDH), and increase mitochondrial membrane potential (MMP) and expressions of peroxisome proliferator-activated receptor gamma coactivator- $1 \alpha$ (PGC$1 \alpha$ ) and nuclear factor erythroid 2-related factor $2(\mathrm{Nrf2})$ in H9C2 cells suffered from oxygen-glucose deprivation (OGD) [11].

Inhibition of mitochondrial permeability transition pore (MPTP) opening can protect heart from ischemia/reperfusion (I/R) injury. The protective effect of HSYA on the myocardial ischemia/reperfusion $(\mathrm{MI} / \mathrm{R})$ rats and the ventricular myocytes isolated from those animals could be attenuated by a MPTP opener called atractyloside and a restrainer of nitric oxide synthase (NOS) named L-NAME [12]. Meanwhile, in the isolated cardiac myocytes stimulated by anoxia/reoxygenation or ionomycin, HYSA increased rod shape cells in the closed MPTP condition and decreased round cells with open MPTP [13]. Another two targets of HYSA against MI or MI/R damage are hemeoxygenase-1 (HO-1) and hemeoxygenase-2 (HO-2). HSYA was able to promote neovascularization and cardiac function recovery in vivo and in vitro by acting through the HO-1/VEGFA/stromal cell derived factor-1 (SDF-1 $\alpha$ ) cascade [14]. In hypoxia/reperfusion- (H/R-) induced H9C2 cells, HYSA exhibited antiapoptotic and antioxidative effects by mediating the protein kinase $\mathrm{B}(\mathrm{Akt}) / \mathrm{Nrf} 2 / \mathrm{HO}-1$ signaling pathway [10, 15]. Jia et al. [16] also reported that HSYA could significantly increase HO-2 expression, adenosine triphosphate (ATP) level, and Mn-superoxide dismutase (SOD) activity and restrain cytochrome c (cyto c) release and MDA level in H9C2 cells suffered from $H / R$, and this cardioprotective property of HSYA was mainly mediated via the phosphoinositide 3-kinase (PI3K)/Akt/HO-2 pathway independent of extracellular regulated protein kinases (ERK)/glycogen synthase kinase- $3 \beta$ (GSK- $3 \beta)$ pathway.

The toll-like receptor 4 (TLR4) signaling pathway may also take part in the protective effect of HSYA. Administration of HSYA inhibited the elevated expression of TLR4 as well as the increased indexes including infarct size, CK-MB, and LDH activity caused by MI/R. Further evidence was that HYSA failed to lessen MI/R damage in the TLR4-knockout mice. Additionally, in neonatal rat ventricular myocytes (NRVMs) subjected to H/R and lipopolysaccharide (LPS), HSYA increased cell viability and downregulated excessive tumor necrosis factor $\alpha$ (TNF- $\alpha$ ) and interleukin-1 $\beta$ (IL-1 $\beta$ ) and overexpressions of TLR4 and nuclear factor-kappa B $(\mathrm{NF}-\kappa \mathrm{B})[17]$.

2.1.3. Antihypertension Effect. Hydroxysafflor yellow A offered the potential for reducing blood pressure. The mean arterial pressure (mAP) and HR (heart rates) in both of the normotensive rats and the spontaneously hypertensive rats (SHR) could be markedly reduced by HSYA [18]. Further study disclosed that HSYA could downregulate the levels of left ventricular systolic pressure (LVSP), left ventricular end-diastolic pressure (LVEDP), and the maximum rate of increase of left ventricular pressure $\left(+\mathrm{d} p / \mathrm{d} t_{\max }\right)$ as well as HR but had little effect on the maximum rate of decrease of left ventricular pressure $\left(-\mathrm{d} p / \mathrm{d} t_{\max }\right)$ in the isolated rat heart [18]. Mechanistically, large conductance $\mathrm{Ca}^{2+}$-activated $\mathrm{K}^{+}$channel $\left(\mathrm{BK}_{\mathrm{Ca}}\right)$ and ATP-sensitive potassium channel $\left(\mathrm{K}_{\mathrm{ATP}}\right)$ was responsible for the effects of HYSA on blood pressure and cardiac function [18]. The test in vitro showed that HSYA could enhance the reduced diastolic response induced by acetylcholine (Ach) and sodium nitroprusside (SNP) and attenuate the vascular contractile effect of PE in the aorta ring isolated from the model [19]. In rat thoracic aorta rings, HSYA inhibited PE-induced endotheliumindependent vasoactive response via inhibiting inositol 1,4,5triphosphate (IP3) receptor in VSMCs and thus reducing extracellular $\mathrm{Ca}^{2+}$ influx and intracellular $\mathrm{Ca}^{2+}$ release [20].

Vascular adventitia proliferation and hyperplasia are of great importance for hypertension occurrence. HSYA has a suppressive effect on rat adventitial fibroblasts proliferation and collagen synthesis stimulated by angiotensin II (Ang II) in vitro. It also inhibited the elevated expressions of matrix metallopeptidase-1 (MMP-1), TGF- $\beta 1, \alpha$-smooth muscle actin ( $\alpha$-SMA), and NF- $\kappa$ B p65 in this model [21]. HYSA inhibited proliferation and dedifferentiation of aorta vascular smooth muscle cells (VSMCs) exposed to platelet-derived growth factor- (PDGF-) BB into a proliferative phenotype, which might be associated with its inhibition of nitrous oxide (NO) and cyclic guanosine monophosphate (cGMP) production, Akt signaling activation, and cycle related proteins as well as its elevation of HO-1 in VSMCs [22]. At the same time, HYSA could suppress proliferation and migration of VSMCs induced by LPS and downregulate levels of TNF- $\alpha$ and interleukin-6 (IL-6) as well as interleukin-8 (IL-8) via the TLR-4/ ras-related C3 botulinum toxin substrate 1 (Rac1)/Akt pathway [23].

HSYA could also act on pulmonary artery. It dosedependently blocked the progression of pulmonary artery remodeling, decreased the cell count in the small pulmonary bronchioles, attenuated right ventricular hypertrophy, and reduced mean right ventricular systolic pressure (mRVSP) in the pulmonary arterial hypertension (PAH) rats induced by hypoxic [24]. In addition, HSYA exerted a vasorelaxing effect on phenylephrine- (PE-) stimulated vascular constrictive action in rat pulmonary artery $(\mathrm{PA})$ rings in a concentrationdependent manner via $\mathrm{Kv}$ activation of pulmonary artery smooth muscle cells (PASMCs) [25]. Further study demonstrated that HYSA could reduce pulmonary arterial hypertension via increasing SOD activity and decreasing levels of MDA and 8-hydroxydeoxyguanosine (8-OHdG) and mRNA of IL- $1 \beta$, IL- 6 , and TNF- $\alpha$ [26].

2.1.4. Effect on Cardiac Hypertrophy. In the overload-induced cardiac hypertrophy rats, HSYA exhibited significantly ameliorative effect on left ventricular mass index (LVMI) induced by the ligation of abdominal aorta, as a consequence of the alleviation of pathological lesion, including smaller cardiac muscle fibers and lightly stained cardiomyocytes nuclei [27]. Additionally, HSYA treatment inhibited cell apoptosis by upregulating $\mathrm{Bcl}-2 / \mathrm{Bax}$ ratio and decreasing matrix metallopeptidase-2 (MMP-2) and MMP-9 levels in serum [27]. 
2.2. Neuroprotective Effects. HYSA offered the therapeutic potential for being natural sources on brain diseases including cerebral ischemia, dementia, Parkinson's disease (PD), and traumatic brain injury (TBI). Previous studies in vivo and in vitro provided further support and evidence for the neurological use of HYSA.

2.2.1. Effect on Cerebral Ischemia. HYSA is considered as a protective agent against cerebral ischemia, which is now a hot research topic of modern medicine. Boring the similar potency to nimodipine, HSYA was found to exert significant neuroprotective effects on the permanent middle cerebral artery occlusion (MCAO) induced focal cerebral ischemic rats as expressed by the reduced neurological deficit scores, infarct area, edema extend, and cell apoptosis [28-30]. The anticerebral ischemic effect of HYSA might result from its suppression of platelet aggregation, thrombin generation, cerebrovascular contraction, cerebrovascular permeability, and thrombin-mediated inflammation as well as promotion of prostacyclin (PGI2)/thromboxane (TXA2) ratio and hemorheology. HYSA could also decrease Ang II, resulting in NF- $\kappa$ B p 65 nuclear translation, p65 binding activity, elevation of ICAM-1 mRNA and protein levels, and neutrophils infiltration $[6,31,32]$.

The neuroprotective actions of HSYA in vivo resulted from several reasons. Chen et al. reported that HSYA critically decreased the apoptosis cell number and increased the Bcl2/Bax proportion in the penumbral cortex of rats subjected to the transient MCAO for $2 \mathrm{~h}$ and followed by $24 \mathrm{~h}$ reperfusion by the PI3K/Akt/GSK-3 $\beta$ pathway [33]. Also, HSYA treatment could cause an increase in the level of brainderived neurotrophic factor (BDNF) in the MCAO mice due to inhibiting the TLR4 signaling pathway [34]. A study by Qi et al. [35] revealed that this effect could be blocked by an Akt inhibitor. The further study indicated that the promotion of HSYA on Akt-autophagy pathway occurred in neuronal-specific cells of penumbra tissue. Additionally, it was found that HYSA inhibited NF- $\kappa$ B pathway and restored the metabolism pathways in the MCAO rats [36].

Blood-brain barrier (BBB), the main shield between cerebral capillaries and brain parenchyma for delivering therapeutic compounds into the brain, shows close relationship with brain ischemic damage. HYSA could penetrate across $\mathrm{BBB}$, downregulate expressions of 12/15-lipoxygenase (12/15LOX) and its metabolite, and trigger decrease of BBB permeability and improvement of tight junction in the MCAO mice via attenuating of occludin, claudin-5, and ZO-1 expressions as well as regulating the tight junction pathway [37-39].

In vitro, HYSA inhibited neurons injury stimulated by glutamate, sodium cyanide $(\mathrm{NaCN})$, and OGD by preventing cell death and $\mathrm{LDH}$ release in cultured rat fetal cortical cells $[28,29]$. Additionally, HSYA alleviated tyrosine nitration induced by authentic peroxynitrite in bovine serum albumin and primary cortical neurons [40]. The further study showed HSYA protected PC12 cells from OGD-induced apoptosis followed by reperfusion through suppressing intracellular oxidative stress and mitochondrial-mediated pathway [41]. In the LPS-activated coexistence system for microglia and neurons, HYSA activated microglia by suppressing TLR4 expression earlier, resulting in later appearance of neuronal apoptosis. The later study showed that the TLR4 pathway played a role in the protective effect of HYSA [42]. Moreover, in cerebral ischemia, release of excessive glutamate always leads to $N$-methyl-D-aspartate receptors (NMDARs) overactivation and excitotoxic neuron injury. Without any effect on the expressions of NR2B-containing NMDARs, HSYA protected rat cortical neurons subjected to $N$-methyl$\mathrm{d}$-aspartate (NMDA) from cell apoptosis via decreasing Bax expression, increasing $\mathrm{Bcl}-2$ expression and downregulating expressions of NR2B-containing NMDARs instead of NR2A-containing NMDARs [43]. Wang et al. further elucidated that HSYA concentration-dependently inhibited excitatory postsynaptic currents (EPSCs) mediated by NMDARs and increased P2/P1 ratio (PPR) of both NMDAR EPSCs and a-amino-3-hydroxy-5-methyl-4-isoxazolepropionic acid receptor (AMPAR) EPSCs resulting in suppressing release of presynaptic transmitter in the mouse hippocampus CA1 region, with a mechanism that HSYA inhibited the membrane depolarization current magnitude induced by NMDARs and ischemic long-term-potentiation (LTP) stimulated by OGD [44]. Moreover, the NMDA-mediated and NMDAR-induced intracellular $\mathrm{Ca}^{2+}$ influx, the NMDAR-induced cell apoptosis and necrotic cell death, and the NMDA-induced mitochondrial injury were inhibited by HYSA in hippocampal neurons [44].

Notably, mitochondria are also likely to be involved in the underlying mechanism. In the cortex mitochondria of rats, HYSA ameliorated the damage induced by cerebral ischemia by inhibiting overloaded $\mathrm{Ca}^{2+}$ and scavenge capability of free radicals, increasing the membrane fluidity and the activities of respiratory enzymes and decreasing the edema degree and the membrane phospholipid decomposability [45]. However, this protective effect would be attenuated by inhibition of the opening of MPTP [46]. And the in vitro study found out that $\mathrm{Ca}^{2+}$ - and $\mathrm{H}_{2} \mathrm{O}_{2}$-stimulated swelling of mitochondria isolated from rat brains was inhibited by HYSA. Meanwhile, HYSA reduced $\mathrm{Ca}^{2+}$ overload-induced ROS generation, improved mitochondrial energy metabolism, and increased ATP level and the respiratory control ratio [47].

2.2.2. Effect on Dementia. Recent findings have discovered the antidementia property of HYSA and provided a foundation for its clinical use in both of vascular dementia (VD) and Alzheimer's disease (AD). HSYA could improve spatial learning and memory in the rat model of $\mathrm{VD}$ via upregulating the expressions of VEGF-A, N-methyl-D-aspartic acid receptor 1 (NR1), BDNF, and NMDAR in the hippocampal, which enhanced LTP and increased synaptic plasticity consequently $[48,49]$. HSYA could significantly reverse cognitive impairment induced by homocysteine (Hcy) in rats, and this was related to attenuation of $\mathrm{A} \beta_{40}$ and $\mathrm{A} \beta_{42}$ levels in hippocampus partially via suppressing PS1 protein level, rescuing apoptosis, and increasing LTP in the AD model [50]. Zhang et al. implied that the protective effect of HYSA on the $\mathrm{A} \beta_{1-42^{-}}$ induced AD mice could be explained by inhibition of inflammation via enhancing the phosphorylation of JAK2/STAT3 pathway $[51,52]$. HYSA also displayed a protective effect from 
neurotoxicity induced by $\mathrm{A} \beta_{25-35}$ in rat pheochromocytoma (PC12) cells by increasing cell viability, stabilizing mitochondrial function, and inhibiting oxidative stress characterized by reduced levels of lactate $\mathrm{LDH}$, intracellular ROS and MDA, and neuronal apoptosis [53].

2.2.3. Effect on Parkinson's Disease. HYSA is also a potential candidate drug for PD, a long-term degenerative disorder. This agent had a neuroprotective role in the 6hydroxydopamine- (6-OHDA-) induced PD rats. It could also increase the levels of dopamine and its metabolites, glial cell line-derived neurotrophic factor (GDNF) and brainderived neurotrophic factor (BDNF) in striatum of $\mathrm{PD}$ rats [58]. Meanwhile, HSYA could effectively relieve motor dysfunction of the PD mice model induced by rotenone and protect dopamine neurons by elevating $\mathrm{TH}$-containing dopaminergic neurons and dopamine content in the striatum in the PD mice. It was suggested that BDNF/tyrosine kinase receptor type $\mathrm{B}(\operatorname{TrkB}) /$ dopamine receptor 3 (DRD3) signaling pathway was responsible for the pharmacological effect of HSYA [59]. Additionally, after coadministration of HSYA with L-DOPA, the 6-OHDA-induced PD rat model exerted the attenuated dyskinesia, the prolonged motor response duration, and the downregulated expression of dopamine $\mathrm{D}$ receptor in the striatum, compared with the PD rats administrated by L-DOPA only [60]. Besides, it was demonstrated that HSYA improved cell viability, reduced cell apoptosis, and increased levels of SOD and glutathione (GSH), the ratio of $\mathrm{Bcl}-2 / \mathrm{Bax}$, and mRNA levels of neuronspecific enolase (NSE) and microtubule-associated protein2 (MAP-2), providing a mechanism of HYSA protecting the differentiation of mesenchymal stem cells (MSCs) against $\beta$ mercaptoethanol (BME) causes oxidation [61].

2.2.4. Effect on Traumatic Brain Injury. The activities of SOD and catalase (CAT), the level of GSH, and the GSH/oxidized glutathione (GSSG) ratio were enhanced while the levels of MDA and GSSG were reduced in the brain of the TBI rats after HSYA treatment [54]. Another report confirmed that HYSA increased activities of mitochondrial ATPase and tissue plasminogen activator (t-PA) and decreased plasma plasminogen activator inhibitor-1 (PAI-1) activity and MMP9 expression in the hippocampus of the TBI rats [55].

2.2.5. Effect on Other Nervous System Diseases. HSYA treatment markedly alleviated lymphostatic encephalopathy- (LE) induced brain injury in rats by dramatically decreasing the neurological scores, attenuating histological changes especially cell apoptosis in the rostral ventrolateral medulla (RVLM) and reducing heart rate variability. Additionally, downregulation of endothelial nitric oxide synthase (eNOS) expression in both of mRNA and protein levels of the RVLM in LE rats were prevented by HYSA [56]. In the spinal cord compression injury rats, HSYA treatment significantly attenuated spinal cord edema and improved motor function outcomes in rats, and the potential mechanism of this action was through ameliorating extent of oxidative stress and preventing release of proinflammatory molecules with inhibition of NF- $\kappa \mathrm{B}$ [57].

2.3. Hepatoprotective Effects. The hepatoprotective activities of HYSA have attracted the attention from the researchers. Recent evidences support HYSA as an antifibrotic agent in hepatic disorders.

2.3.1. Effect on Hepatic Fibrosis. The hepatoprotective effects of HYSA are especially related to its antifibrotic and antioxidative actions. In respect of antihepatic fibrosis assay in vivo, HSYA treatment could not only reduce the serum levels of alanine aminotransferase (ALT), aspartate transaminase (AST), hyaluronan (HA), laminin (LN), and type III procollagen (PC III) as well as the hepatic levels of ROS and MDA but also elevate the activity and mRNA of SOD, glutathione peroxidase (GPx), and expression of TGF- $\beta 1$ in liver tissue of the long-term alcohol-injured rats [62]. The histological studies suggested the alcohol induced liver damage such as hepatic fibrogenesis which could be greatly alleviated by HYSA [62]. Also, HYSA could decrease levels of total cholesterol (TC), triglyceride (TG), and mRNA expressions of transforming growth factor $\beta$ receptor I (TGF $\beta-\mathrm{R}$ I), transforming growth factor $\beta$ receptor (TGF $\beta$-R II), mitogen-activated protein (MAP), ERK, MAP/ERK kinase kinase 3 (MEKK3), and MAP kinase kinase-5 (MEK5) as well as phosphorylation of ERK5 in the tetrachloride- $\left(\mathrm{CCl}_{4}^{-}\right)$induced hepatic fibrosis rats $[63,64]$. The precise mechanism of the hepatoprotective property was that HSYA strengthened expressions of peroxisome proliferator-activated receptor- $\gamma(\operatorname{PPAR} \gamma)$ and MMP-2, downregulated expressions of TGF- $\beta 1$ and TIMP1 , and reduced $\alpha$-SMA level by stimulating PPAR $\gamma$ activity [65]. Meanwhile, the ameliorative effects of HYSA on the $\mathrm{CCl}_{4}$ / high fat diet- (HFD-) stimulated liver fibrosis rats were significantly alleviated by the PPAR $\gamma$ inhibitor, which was a result of blocking p38 MAPK phosphorylation [66]. Hepatic stellate cells (HSCs) appear to be vital in the development of liver fibrosis [67]. The testing in vitro suggested proliferation of HSCs stimulated by $\mathrm{H}_{2} \mathrm{O}_{2}$ was inhibited by HSYA because of HSYA's blockage of the cell cycle from $G_{0} / G_{1}$ to $G_{1} / S$ [65]. Moreover, in HSCs, HYSA also inhibited cell proliferation and induced cell apoptosis in a dose- and time-dependent fashion accompanying with decreasing expressions of type I alpha collagen (Col I $\alpha$ ), $\alpha$-SMA and type III alpha collagen (Col III $\alpha), \mathrm{Bcl}-2$, and myocyte enhancer factor 2C (MEF2C) and increasing expressions of cyto $c$, Caspase-9, and Caspase3 , which resulted from the activation of the extracellular regulated protein kinases (ERK5) pathway as well as the extracellular regulated protein kinases 1/2 (ERK1/2) pathway $[68,69]$.

2.3.2. Effect on Hepatic Ischemia. HSYA reduced serum AST and ALT levels and expressions of TNF- $\alpha$ and IL$1 \beta$, ameliorated inflammation and necrosis, and blocked macrophage recruitments in the I/R mice. Similarly, in vitro, pretreatment of HYSA resulted in the weakened migratory reaction and the reduced inflammatory cytokines in the H/Rchallenged RAW264.7. The further study showed that HSYA 
had suppressive effect on expressions of MMP-9 and ROS, $\mathrm{NF}-\kappa \mathrm{B}$ activation, and $\mathrm{p} 38$ phosphorylation in the injured RAW264.7 cells [70].

2.4. Pulmonary Protective Effect. The various researches exhibited remarkable pulmonary protective properties of HYSA in vivo and in vitro, especially about the inhibitory effect on chronic obstructive pulmonary disease (COPD), acute lung injury (ALI), and lung fibrosis.

\subsubsection{Effect on Airway Inflammation. HSYA treatment could} attenuate the airway hyperresponsiveness (AHR) cell chemotaxis, proinflammatory cytokines, type 2 helper T cell 2 (Th2) cytokines, total and ovalbumin- (OVA-) specific IgE and adhesion molecules in bronchoalveolar lavage fluid (BALF), and inflammatory responses levels in the lung tissue of the OVA-induced asthmatic mice. Study suggested that HSYA treatment inhibited the transcriptional activity of NF- $\kappa \mathrm{B}$ by inhibiting NF- $\kappa \mathrm{B}$ p 65 nuclear translocation and nuclear factor of $\mathrm{I} \kappa \mathrm{B}-\alpha$ phosphorylation and degradation [71]. Wang et al. reported that HSYA markedly suppressed the thickening and collagen deposition of the small airway and weakened TGF- $\beta 1$ mRNA and protein expression as well as type I collagen (Col I) and $\alpha$-SMA expressions in the lung of the COPD model in the rats stimulated by cigarette smoke and LPS. In addition, HSYA elicited inhibitory effect on phosphorylation of p38 mitogen-activated protein kinase (MAPK) in the rat lung tissue [72].

2.4.2. Effect on Acute Lung Injury. HSYA could alleviate pulmonary edema, reduce acidosis, increase partial pressure of oxygen $\left(\mathrm{PaO}_{2}\right)$, and inhibit inflammatory cell infiltration, lung mRNA expressions of TNF- $\alpha$ and ICAM-1, and levels of plasma IL- 6 and IL-1 $\beta$ [73]. HSYA significantly increased the activities of antioxidant enzymes, inhibited the inflammatory response via the cAMP/PKA pathway activation, and attenuated OA-induced lung injury [74]. Additionally, HSYA decreased lung permeability, platelet count, and ADP-mediated platelet aggregation as well as cytokine levels in serum and BALF in the aged rats with gasoline engine-induced lung injury. Moreover, it suppressed overexpressions of ICAM-1, vascular cell adhesion molecule1 (VCAM-1), and proinflammatory cytokines in platelets and lung tissue. And decrease in cAMP level in lung and platelets and PKA activity and PPAR $\gamma$ expression in platelets induced by gasoline engine exhaust were also reversed by HYSA [75]. Meanwhile, in the sepsis-associated ALI mice, the inflammatory infiltration and proinflammatory cytokine expressions in lung, pneumochysis, and respiratory insufficiency induced by LPS were greatly relieved by HYSA treatment. Its suppression of $\mathrm{p} 38 \mathrm{MAPK}$ phosphorylation as well as NF- $\kappa \mathrm{B}$ activation was responsible for this protective effect [76]. Also, HSYA ameliorated the pathological state and lung vascular permeability of the LPS-induced ALI in mice. This effect was accompanied with the negative effect on pulmonary myeloperoxidase (MPO) activity and levels of serum TNF- $\alpha$, IL- $1 \beta$, IL-6, and interferon- $\beta$ (IFN- $\beta$ ). The further investigation demonstrated that intraperitoneal injection of LPS to the mice resulted in upregulation of protein expressions of TLR4, myeloid differentiation factor 88 (MyD88) and Toll/IL-1 receptor- (TIR-) domain-containing adapter-inducing interferon- $\beta$ (TRIF) and phosphorylation of MAPKs, translocation of NF- $\kappa \mathrm{B} / \mathrm{p} 65$, and downregulation of I $\kappa \mathrm{B}-\alpha$, all of which could be deteriorated by HSYA [77]. Zhang et al. observed the negative influence of HSYA on the binding of LPS to the cell membrane receptor in the LPS-caused ARDS mice model [78]. And injection of HYSA alleviated the mRNA and protein levels of Col I, type III collagen (Col III), $\alpha$-SMA, myeloid differentiation-2 (MD-2), and cluster of differentiation 14 (CD14) as well as inflammatory factors in plasma or lung and the collagen deposition in lung via suppressing the TLR4/NF- $\kappa \mathrm{B}$ pathway. The in vivo test demonstrated the inhibitory effect of HYSA on the specific binding of LPS to receptors on A549 or Eahy926 cell membranes, suggesting the TLR4 receptor a target of HSYA on the cell membrane [78].

2.4.3. Effect on Lung Fibrosis. Injection of HYSA attenuated the pathologic changes of pulmonary inflammation, increased the body weight, $\mathrm{PaO}_{2}$ and decreased $\mathrm{PaCO}_{2}$, mRNA expressions of TNF- $\alpha$, IL- $1 \beta$, TGF- $\beta 1$, and IL-6, MDA activity, and the count of NF- $\kappa \mathrm{B}$ p 65 positive cells in the BLM-injured acute inflammation rats. This protective effect of HYSA might be due to inhibition of NF- $\kappa \mathrm{B}$ activation and p38 MAPK phosphorylation in lung tissue $[79,80]$. Moreover, 21 days of HSYA to the BLM-induced chronic pulmonary fibrosis rats resulted in fibrosis amelioration by decreasing collagen deposition, and mRNA expression of TGF- $\beta 1, \alpha-$ SMA and Col I connective tissue growth factor (CTGF) as well as $\alpha$-SMA level [80, 81].

Assayed with human alveolar epithelial A549 cells, HYSA was found to inhibit Smad3 phosphorylation and Col I mRNA expression stimulated by TGF- $\beta 1$ [81]. And HSYA exhibited inhibitory effects on LPS-induced inflammatory response in A549 cells by suppressing myeloid differentiation factor 88 (MyD88), TLR-4, TNF- $\alpha$, ICAM-1, IL-1 $\beta$, and IL6 at the mRNA and protein expression levels and leukocytes adhesion to A549 cells. The mechanism was due to its negative regulation of $\mathrm{NF}-\kappa \mathrm{B}$ p 65 nuclear translocation and p38 MAPK phosphorylation [82]. Meanwhile, TGF- $\beta 1-$ induced alteration in proliferation, migration, extracellular matrix (ECM) accumulation, and degradation of human fetal lung fibroblasts MRC- 5 were inhibited by HYSA. Further study disclosed that HSYA blocked the binding of TGF- $\beta 1$ to the cytoplasmic receptors of TGF- $\beta 1$-stimulated MRC5 including TGF $\beta$-R II and suppressed the lung pathological alteration and $\alpha$-SMA, Col I $\alpha 1$, and $\mathrm{FN}$ expressions as well as phosphorylation of mothers against decapentaplegic homolog 2 (Smad2), mothers against decapentaplegic homolog 3 (Smad3) and ERK, nuclear translocation of Smad2 and Smad3, and the binding of Smad3 to Col I promoter $[83,84]$.

2.5. Antitumor Effects. Several reports have provided strong evidences that HYSA is valuable for oncotherapy. HYSA was considered as a natural compound which could inhibit 
multistage carcinogenesis processes such as progression, adhesion, invasion, and migration. In HYSA-treated MCF7 cells, Li et al. [85] observed increased cell apoptosis and ROS level, upregulated expressions of Bax and p53, blocked cell cycle, downregulated Bcl-2 and cyclin D1, released cyto $c$, activated Caspase-3, and disrupted MPP with the mechanism of its negative regulation on the NF- $\kappa \mathrm{B} / \mathrm{p} 65$ nuclear translocation. Also, HSYA, acting as a PPAR $\gamma$ agonist, showed inhibitory effect on proliferation and cell cycle transition and stimulatory effect on cell apoptosis in BGC-823 cells [86]. As for abnormally proliferated HUVECs cultured in HepG2 cell cultural supernatant, HSYA suppressed the expressions of vascular endothelial growth factor (VEGF) and its kinase insert domain receptor (KDR) through the Ras-Raf-MEKERK1/2 signaling pathway and dephosphorylated the corresponding kinase molecules [87]. Moreover, HYSA exerted inhibitory effect on cancer cell growth of tumor-bearing mice. This effect was associated with its downregulation of angiogenesis. Xi et al. [88] proved that it reduced the microvessel count and microvessel density of the transplanted tumors in the BGC-823 cell bearing mice while Yang et al. [89] reported this drug had the advantage of decreasing VEGFA, basic fibroblast growth factor (bFGF), vascular endothelial growth factor receptor 1 (VEGFR1), and mRNA expression levels of cyclin D1, c-myc, and c-fos in the BGC-823 tumorbearing mice through suppressing the ERK/MAPK and the NF- $\kappa$ B pathway. Additionally, along with the ability of inhibiting the process of SMMC-7721 covering proliferation, adhesion, invasion, and migration, HYSA could suppress pulmonary metastasis of liver cancer. In the pulmonary metastatic mouse model of $\mathrm{H} 22$ cells, the formation of a complex with E-cadherin/ $\beta$-catenin induced by HYSA could activate the expression of PPAR $\gamma$ and inhibit the activity of MMP-2, finally leading to decrease in degradation of ECM and suppression of epithelial-mesenchymal transition (EMT) [90]. In addition, HSYA could reversibly and noncompetitively inhibit of human recombinant aldehyde dehydrogenase $1(\mathrm{ALDH} 1)(\mathrm{Ki}=0.267 \pm 0.024 \mathrm{mM})$ indicating it as a potential agent for treating ALDH1-associated cancers [91].

2.6. Metabolic Regulation Effect. The quantity of preadipocytes, adipocytes differentiation, and lipid accumulation play a key role in lipid metabolism [92]. HSYA significantly time- and dose-dependently prohibited the proliferation of 3T3-L1 cells. This effect was accompanied by a decline in the amount of intracellular lipid and triglyceride (TG) and a rise in mRNA expression of hormone-sensitive lipase (HSL) and promoter activities [93]. HSYA competitively inhibited $\alpha$ glucosidase in a reversible way with $I C_{50}=1.1 \pm 0.22 \mathrm{mM}$ and $\mathrm{Ki}=1.04 \pm 0.23 \mathrm{mM}$, respectively. HSYA-induced structural change of $\alpha$-glucosidase was mainly regional unfolding [94]. Advanced glycation end products (AGEs) and methylglyoxal (MGO) accumulation usually appear in individuals with diabetes and cause the occurrence of vascular complication [95]. Ni et al. [96] demonstrated that MGO-induced bovine serum albumin (BSA) glycation could be inhibited by HYSA. Besides, HSYA showed significantly inhibitory effect on glucose- (GLU-) induced development of AGEs formation and $N$-acetyl-glycyl-lysine methyl Ester (G.K.) peptide-mediated ribose glycation. Also, HSYA could defend against MGO-induced damage in cultured human brain microvascular endothelial cells (HBMEC) by increasing cell viability and decreasing cell mortality. It suppressed cell apoptosis and Caspase-3 expression in HBMEC and inhibited AGEs accumulation in HBMEC after treated with MGO [97].

2.7. Endothelium Cell Protection. In vivo, HSYA effectively recovered perfusion of ischemic hindlimb tissue and gave a rise of the arteriole and capillary densities in the femoral artery-interrupted ischemic gastrocnemius muscles of the mice [98]. Ox-LDL-caused endothelial injury could be relieved by HSYA dose-dependently. Proteomic investigation revealed that this effect was related to the antiapoptotic activity of voltage dependent anion-selective channel 2 (VDAC2) [99]. Moreover, HSYA promoted the viability of LPS-injured HUVEC. It also inhibited the subsequent inflammation induced by LPS in HUVEC including NF- $\kappa \mathrm{B}$ p65 subunit DNA binding, I $\kappa \mathrm{B} \alpha$ phosphorylation, ICAM-1, and E-selectin mRNA levels elevation and phosphorylation of p38 MAPK or c-Jun N-terminal kinase (JNK) MAPK, cell surface ICAM-1 protein expression, and leukocyte adhesion to HUVEC [100]. Meanwhile, HYSA elicited a protective effect on HUVECs from hypoxia by attenuating cell apoptosis and cell cycle arrest, which was the result of the upregulation of the $\mathrm{Bcl}-2 / \mathrm{Bax}$ ratio and the hypoxia inducible factor- $1 \alpha$ (HIF-1 $\alpha-$ ) VEGF pathway as well as NO contend and eNOS expression and downregulation of p53 protein expression [101]. However, with little effect on the normal HUVEC, HSYA caused an increase of the capillary-like tube formation and migration of HUVEC, which could be reversed by an anti-Tie-2 neutralizing antibody. Expression of angiopoietin 1 and phosphorylation of Tie2, Akt, and ERK $1 / 2$ were greatly elevated by HSYA [102].

2.8. Other Effects. As an anti-inflammatory molecule, HYSA could inhibit LPS-induced NLRP3 inflammasome activation via binding to Xanthine Oxidase in mouse RAW264.7 macrophages [103]. HSYA-mediated sonodynamic therapy could induce ROS-dependent autophagic response via the $\mathrm{PI} 3 \mathrm{~K} / \mathrm{Akt} / \mathrm{mTOR}$ signaling pathway in THP-1 macrophages [104]. Besides, HSYA inhibited rabbit polymorphonuclear (PMN) activation induced by LPS by reducing LPS-induced elevated adhesion potency, free calcium concentration, TNF$\alpha$ and IL- 6 mRNA expression, and NF- $\kappa$ B p65 nuclear translocation [105]. Also, HYSA exhibited anti-inflammatory effect as evidenced by inhibiting the IL- $1 \beta$-induced release of IL-6, IL-8, and MMP-1 in SW982 human synovial cell, and this was associated with suppression of ERK, NF- $\kappa \mathrm{B}$, and activator protein-1 (AP-1) signaling [106]. And HSYA could reduce strike-triggered local oedema and neutrophil infiltration in skeletal muscle via inhibiting p38 MAPK phosphorylation and suppressing NF- $\kappa \mathrm{B}$ pathway activation [107]. It was also an effective therapeutic agent in ameliorating sepsis-induced apoptosis of cluster of differentiation $4+$ (CD4+) T lymphocytes through its anti-inflammatory and antiapoptotic effects [108]. 


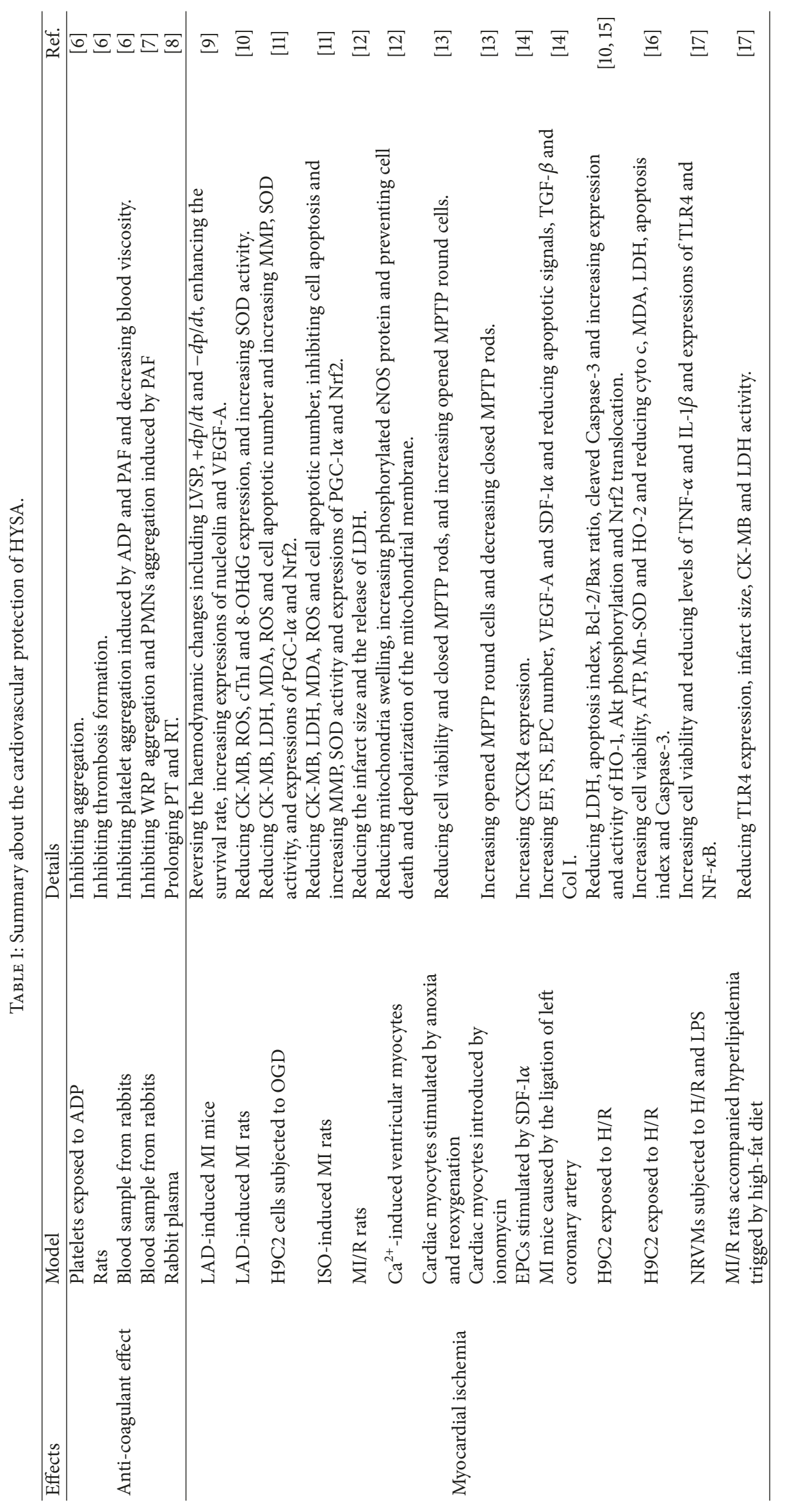




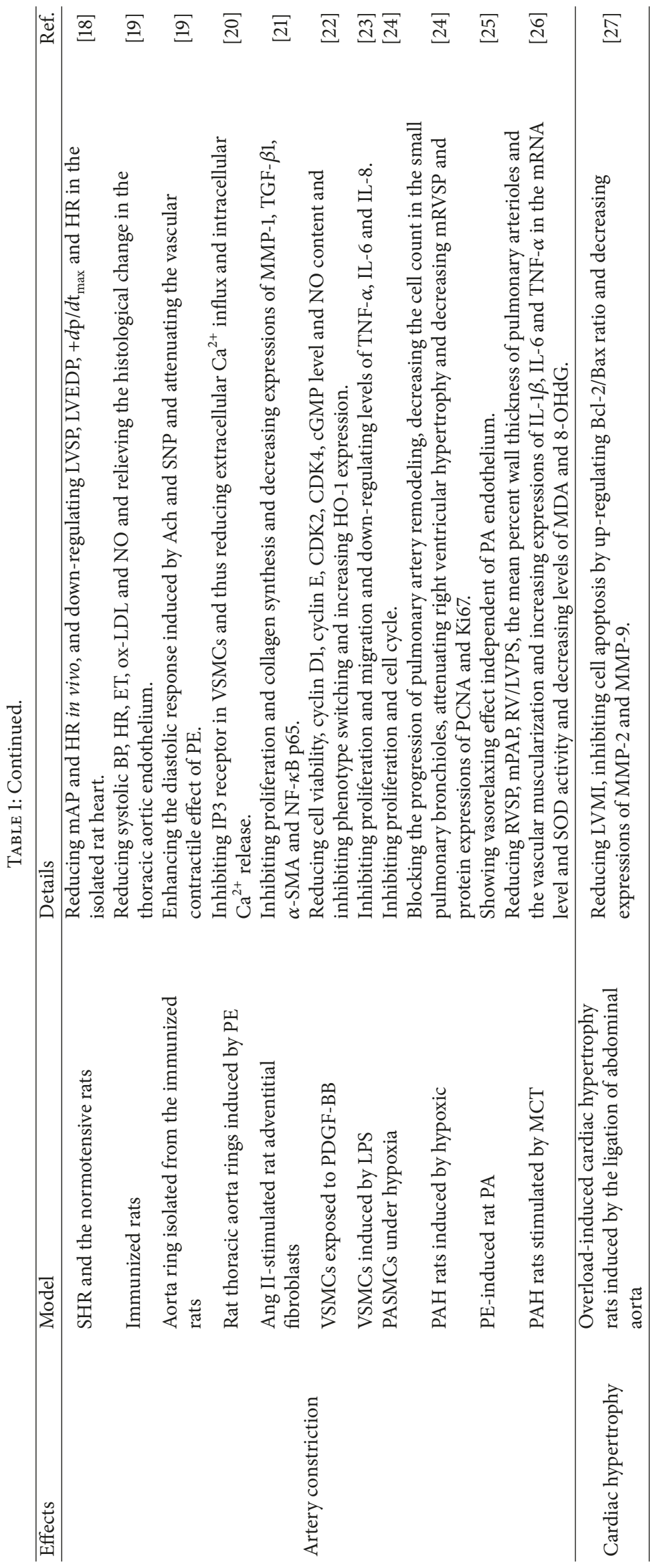




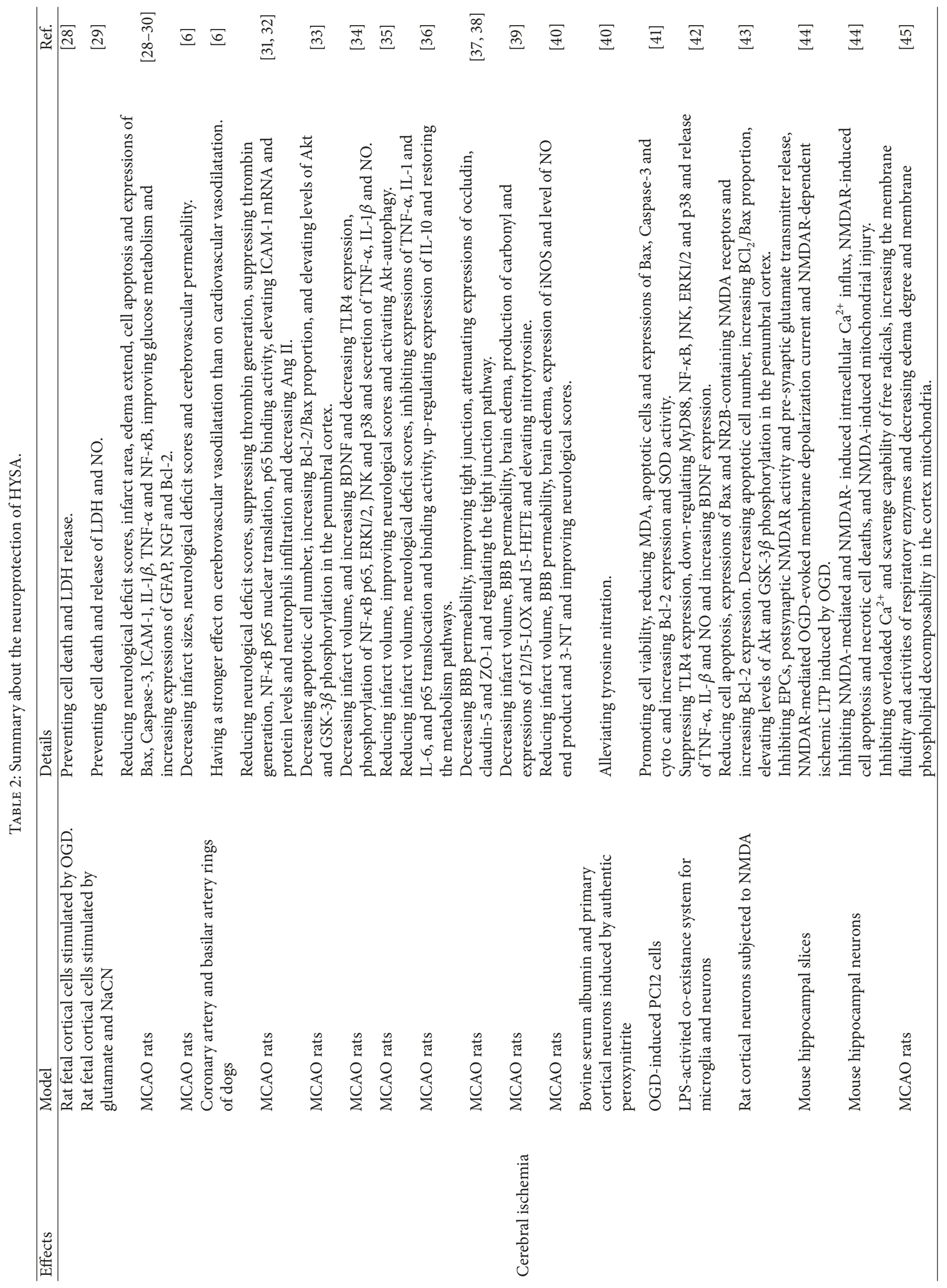




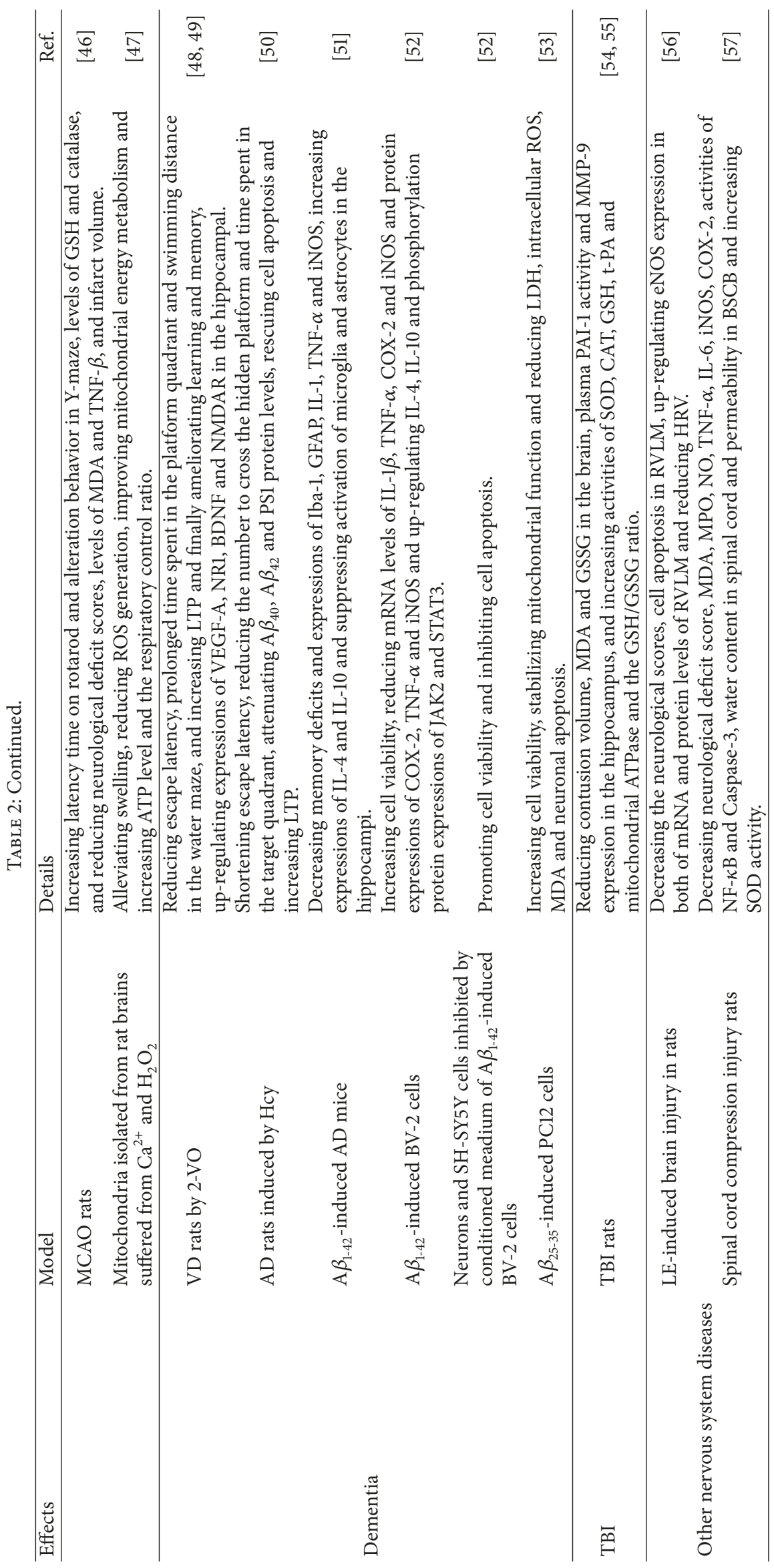




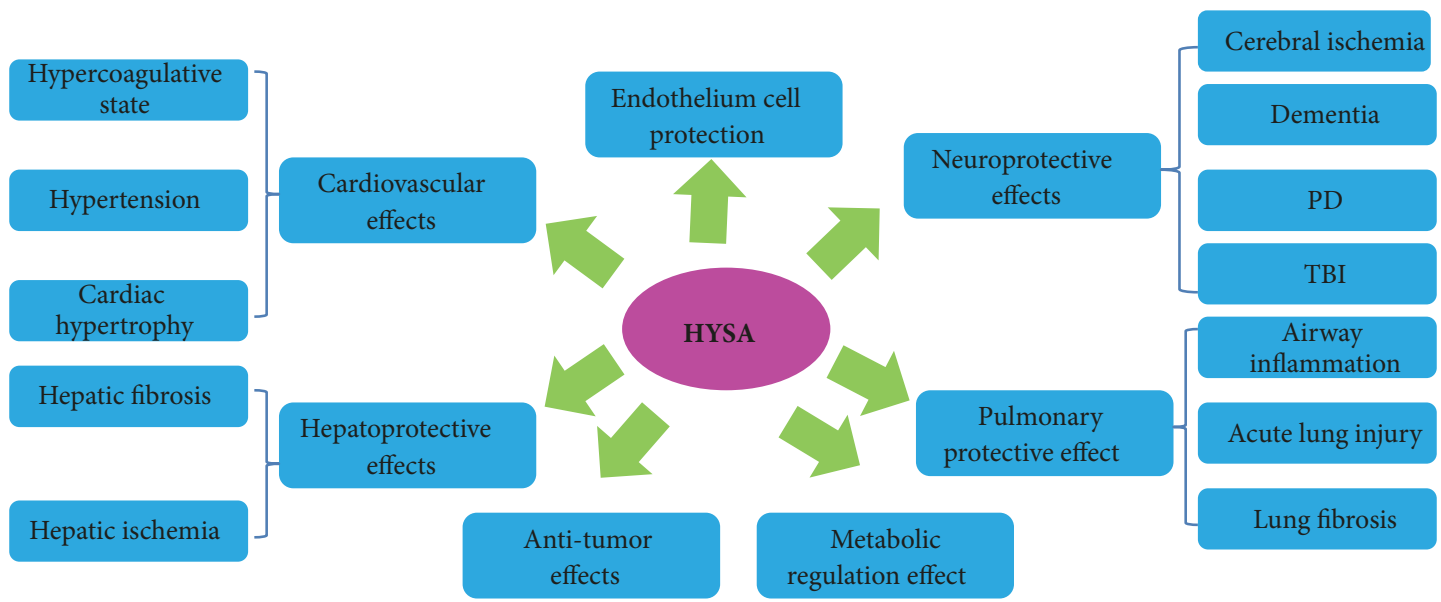

FIGURE 2: Pharmacological effects of HYSA.

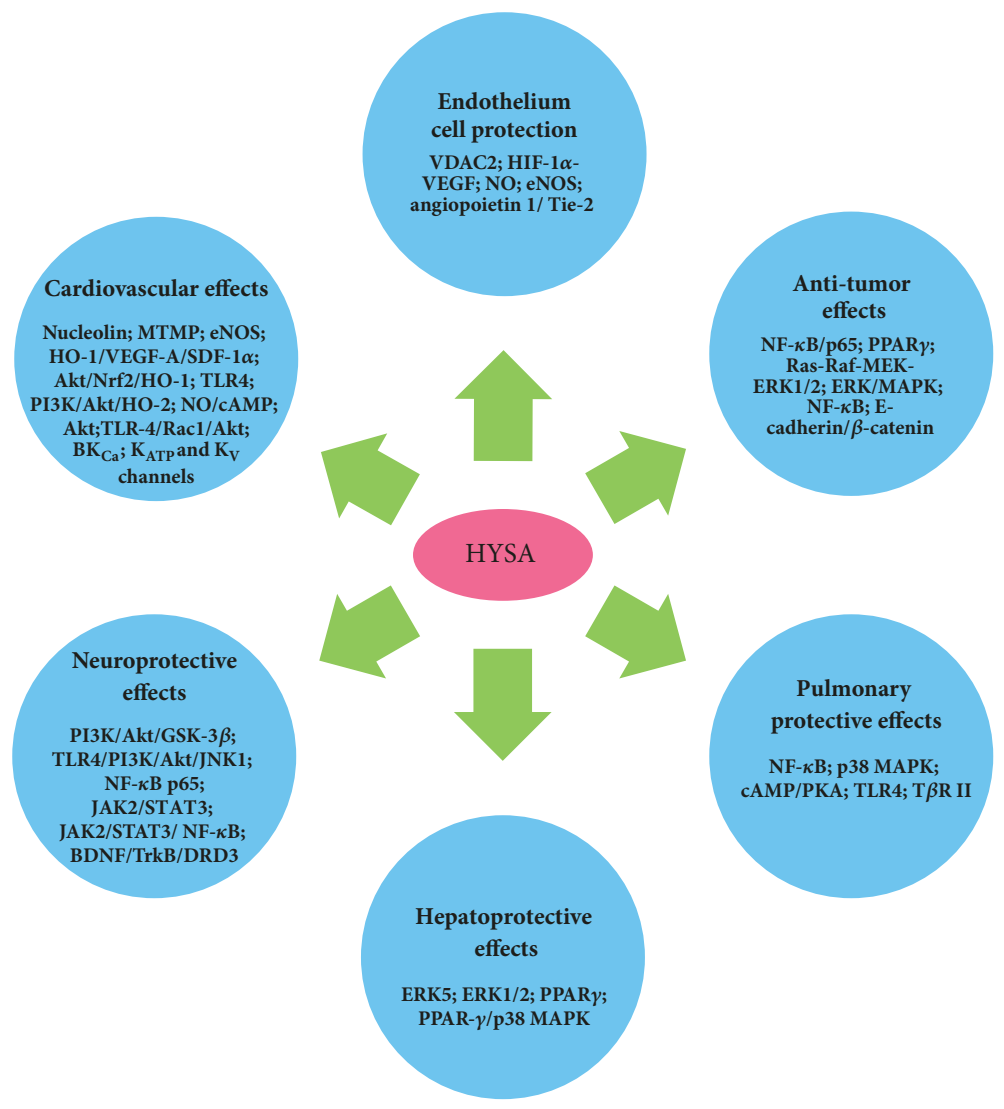

FIGURE 3: Action mechanism and corresponding mediators of HYSA.

HSYA had protective effect against fibrosis in renal cells, through inhibiting TGF- $\beta 1 /$ Smad3-mediated epithelialmesenchymal transition signaling pathway [109]. Topical use of HSYA could relieve the UV-induced skin damage in mice by promoting recovery from stretching, inhibiting epidermal hyperproliferation and keeping the structural integrity of the skin via antioxidative activity [110]. Moreover, HSYA could strongly inhibit tyrosinase by binding and changing the tertiary structure of tyrosinase [111].

\section{Conclusion}

Noncommunicable diseases (NCDs), namely, heart disease, stroke, cancer, diabetes, and chronic lung disease, are causing worldwide public health problem and alarmingly leading causes of almost $70 \%$ deaths. Natural chemicals are favorable resources that may be utilized to develop such agents. HYSA, a component isolated from safflower with little toxicity [112], showed various pharmacological effects in vitro and in vivo 
(summarized in Figure 2), including cardiovascular protection (summarized in Table 1), neuroprotection (summarized in Table 2), metabolism regulation, antitumor effect, and liver, lung, and EC protective activities. These activities indicate the use of HYSA for prevention and/or the treatment of NCDs and other intractable diseases such as AD, PD, TBI, ALI, and fibrotic diseases. Although HYSA possesses extensive pharmacological effects, it is currently used as a drug mainly in treating cardiovascular and cerebrovascular diseases. It needs to be clinically explored in other aspects especially for respiratory, hepatic and metabolic diseases, and malignancy. Recent studies focusing on various bioactivities of HYSA have yielded promising results, demonstrating both the pharmacological effects and the molecular mechanisms of HYSA (summarized in Figure 3). Further investigation of the molecular mechanisms of HYSA is anticipated to expand the clinical applications of HYSA.

\section{Conflicts of Interest}

The authors declared that there are no conflicts of interest.

\section{Authors' Contributions}

Hui Ao and Wuwen Feng contributed equally to this paper.

\section{Acknowledgments}

This work was supported by funds from Sichuan Youth Scientific and Technological Innovation Research Team (2017TD0001) and the State Key Laboratory Breeding Base of Systematic Research, Development and Utilization of Chinese Medicine Resources.

\section{References}

[1] Z. Ekin, "Resurgence of safflower (Carthamus tinctorius L.) utilization: A global view," Journal of Agronomy, vol. 4, no. 2, pp. 1812-5379, 2005.

[2] M. R. Meselhy, S. Kadota, Y. Momose et al., "Two new quinochalcone yellow pigments from carthamus tinctorius and $\mathrm{Ca}^{2+}$ antagonistic activity of tinctormine," Chemical \& Pharmaceutical Bulletin, vol. 41, no. 10, pp. 1796-1802, 1993.

[3] Pharmacopoeia Commission of People's Republic of China, Pharmacopoeia of People's Republic of China, 2015 edition, Chinese Medicine Science and Technology Publishing House, Beijing, China, 2015.

[4] J. H. Li, G. L. Xu, S. M. Lin et al., "Systematic evaluation of effectiveness and safety on Safflower Injection in angina pectoris," Journal of Emergency in Traditional Chinese Medicine, vol. 21, no. 6, pp. 932-939, 2012.

[5] H. J. Cui, H. Y. He, and Z. H. Xing, "Meta-analysis of Safflower Yellow Injection for the treatment of the overall effectiveness power and safety of acute cerebral infarction," China Traditional Patent Medicine, vol. 33, no. 8, pp. 1299-1302, 2011.

[6] Y. Sun, D.-P. Xu, Z. Qin et al., "Protective cerebrovascular effects of hydroxysafflor yellow A (HSYA) on ischemic stroke," European Journal of Pharmacology, vol. 818, pp. 604-609, 2018.
[7] B. X. Zang, M. Jin, N. Si et al., "Antagonistic effect of hydroxysafflor yellow A on the platelet activating factor receptor," Acta Pharmaceutica Sinica, vol. 37, no. 9, pp. 696-699, 2002.

[8] B. X. Zang, J. Min, and L. Jinrong, "Study on the anti-coagulant effect of Hydroxysafflor yellow A," Chinese Traditional and Herbal Drugs, vol. 38, no. 5, pp. 741-743, 2007.

[9] J. Zou, N. Wang, M. Liu et al., "Nucleolin mediated proangiogenic role of Hydroxysafflor Yellow A in ischaemic cardiac dysfunction: Post-transcriptional regulation of VEGF-A and MMP-9," Journal of Cellular and Molecular Medicine, vol. 22, no. 5, pp. 2692-2705, 2018.

[10] T. Hu, G. Wei, M. Xi et al., "Synergistic cardioprotective effects of Danshensu and hydroxysafflor yellow A against myocardial ischemia-reperfusion injury are mediated through the Akt/Nrf2/HO-1 pathway," International Journal of Molecular Medicine, vol. 38, no. 1, pp. 83-94, 2016.

[11] M. Chen, M. Wang, Q. Yang et al., "Antioxidant effects of hydroxysafflor yellow A and acetyl-11-keto- $\beta$-boswellic acid in combination on isoproterenol-induced myocardial injury in rats," International Journal of Molecular Medicine, vol. 37, no. 6, pp. 1501-1510, 2016.

[12] Y.-N. Liu, Z.-M. Zhou, and P. Chen, "Evidence that hydroxysafflor yellow A protects the heart against ischaemia-reperfusion injury by inhibiting mitochondrial permeability transition pore opening," Clinical and Experimental Pharmacology and Physiology, vol. 35, no. 2, pp. 211-216, 2008.

[13] G. A. Huber, S. M. Priest, and T. P. Geisbuhler, "Cardioprotective effect of hydroxysafflor yellow A via the cardiac permeability transition pore," Planta Medica, vol. 84, no. 08, pp. 507-518, 2018.

[14] G. Wei, Y. Yin, J. Duan et al., "Hydroxysafflor yellow A promotes neovascularization and cardiac function recovery through $\mathrm{HO}$ 1/VEGF-A/SDF-1 $\alpha$ cascade," Biomedicine \& Pharmacotherapy, vol. 88, pp. 409-420, 2017.

[15] S. X. Liu, Y. Zhang, Y. F. Wang et al., "Upregulation of heme oxygenase-1 expression by hydroxysafflor yellow A conferring protection from anoxia/reoxygenation-induced apoptosis in H9c2 cardiomyocytes," International Journal of Cardiology, vol. 160, no. 2, pp. 95-101, 2012.

[16] J. Min and C. Wei, "Hydroxysafflor yellow A cardioprotection in ischemia-reperfusion (I/R) injury mainly via Akt/hexokinase II independent of ERK/GSK-3 $\beta$ pathway," Biomedicine \& Pharmacotherapy, vol. 87, pp. 419-426, 2017.

[17] D. Han, J. Wei, R. Zhang et al., "Hydroxysafflor yellow A alleviates myocardial ischemia/reperfusion in hyperlipidemic animals through the suppression of TLR4 signaling," Scientific Reports, vol. 6, no. 1, Article ID 35319, 2016.

[18] P.-H. Nie, L. Zhang, W.-H. Zhang, W.-F. Rong, and J.-M. Zhi, "The effects of hydroxysafflor yellow A on blood pressure and cardiac function," Journal of Ethnopharmacology, vol. 139, no. 3, pp. 746-750, 2012.

[19] Z. Jin, W. Zhang, W. Chai, Y. Zheng, and J. Zhi, "Antibodies against AT1 receptors are associated with vascular endothelial and smooth muscle function impairment: protective effects of hydroxysafflor yellow A," PLoS ONE, vol. 8, Article ID e67020, 2013.

[20] L. Zhang, P. H. Nie, G. H. Zhang et al., "Endotheliumindependent vasodilation effect of hydroxysafflor yellow A in thoracic aorta of Wistar rats," Journal of Medicinal Plants Research, vol. 5, no. 11, pp. 2187-2191, 2011.

[21] W. Yuan, D. Yang, X. Sun et al., "Effects of hydroxysafflor yellow A on proliferation and collagen synthesis of rat vascular 
adventitial fibroblasts induced by angiotensin II," International Journal of Clinical and Experimental Pathology, vol. 7, no. 9, pp. 5772-5781, 2014.

[22] Y. Song, L. Long, N. Zhang, and Y. Liu, "Inhibitory effects of hydroxysafflor yellow A on PDGF-BB-induced proliferation and migration of vascular smooth muscle cells via mediating Akt signaling," Molecular Medicine Reports, vol. 10, no. 3, pp. 1555-1560, 2014.

[23] G. Yang, X. Zhou, T. Chen et al., "Hydroxysafflor yellow A inhibits lipopolysaccharide-induced proliferation and migration of vascular smooth muscle cells via Toll-like receptor-4 pathway," International Journal of Clinical and Experimental Medicine, vol. 8, no. 4, pp. 5295-5302, 2015.

[24] L. Li, P. Dong, C. Hou et al., "Hydroxysafflor yellow A (HSYA) attenuates hypoxic pulmonary arterial remodelling and reverses right ventricular hypertrophy in rats," Journal of Ethnopharmacology, vol. 186, no. 20, pp. 224-233, 2016.

[25] Y. Bai, P. Lu, C. Han et al., "Hydroxysafflor yellow A (HSYA) from flowers of carthamus tinctorius L. and its vasodilatation effects on pulmonary artery," Molecules, vol. 17, no. 12, pp. 1491814927, 2012.

[26] X. Han, Y. Zhang, Z. Zhou, X. Zhang, and Y. Long, "Hydroxysafflor yellow A improves established monocrotaline-induced pulmonary arterial hypertension in rats," Journal of International Medical Research, vol. 44, no. 3, pp. 569-584, 2016.

[27] J. Wang, Q. Zhang, X. Mei, and X. Zhang, "Hydroxysafflor yellow A attenuates left ventricular remodeling after pressure overload-induced cardiac hypertrophy in rats," Pharmaceutical Biology, vol. 52, no. 1, pp. 31-35, 2014.

[28] H. Zhu, Z. Wang, C. Ma et al., "Neuroprotective effects of hydroxysafflor yellow A: in vivo and in vitro studies," Planta Medica, vol. 69, no. 5, pp. 429-433, 2003.

[29] S.-Y. Ye and W.-Y. Gao, "Hydroxysafflor yellow a protects neuron against hypoxia injury and suppresses inflammatory responses following focal ischemia reperfusion in rats," Archives of Pharmacal Research, vol. 31, no. 8, pp. 1010-1015, 2008.

[30] L. Deng, H. Wan, H. Zhou, L. Yu, and Y. He, "Protective effect of hydroxysafflor yellow A alone or in combination with acetylglutamine on cerebral ischemia reperfusion injury in rat: A PET study using 18F-fuorodeoxyglucose," European Journal of Pharmacology, vol. 825, pp. 119-132, 2018.

[31] X. Sun, X. Wei, S. Qu, Y. Zhao, and X. Zhang, "Hydroxysafflor yellow A suppresses thrombin generation and inflammatory responses following focal cerebral ischemia-reperfusion in rats," Bioorganic \& Medicinal Chemistry Letters, vol. 20, no. 14, pp. 4120-4124, 2010.

[32] H. B. Zhu, L. Zhang, Z. H. Wang et al., "Therapeutic effects of hydroxysafflor yellow A on focal cerebral ischemic injury in rats and its primary mechanisms," Journal of Asian Natural Products Research, vol. 7, no. 4, pp. 607-613, 2005.

[33] L. Chen, Y. Xiang, L. Kong et al., "Hydroxysafflor yellow a protects against cerebral ischemia-reperfusion injury by antiapoptotic effect through PI3K/Akt/GSK3 $\beta$ pathway in rat," Neurochemical Research, vol. 38, no. 11, pp. 2268-2275, 2013.

[34] Y. Lv, Y. Qian, L. Fu, X. Chen, H. Zhong, and X. Wei, "Hydroxysafflor yellow A exerts neuroprotective effects in cerebral ischemia reperfusion-injured mice by suppressing the innate immune TLR4-inducing pathway," European Journal of Pharmacology, vol. 769, pp. 324-332, 2015.

[35] Z. Qi, F. Yan, W. Shi et al., "AKT-Related autophagy contributes to the neuroprotective efficacy of hydroxysafflor yellow A against ischemic stroke in rats," Translational Stroke Research, vol. 5, no. 4, pp. 501-509, 2014.

[36] Yuanyan Liu, Zeqin Lian, Haibo Zhu et al., "A systematic, integrated study on the neuroprotective effects of hydroxysafflor yellow A revealed by (1)H NMR-based metabonomics and the NF- $\kappa \mathrm{B}$ pathway," Evidence-Based Complementary and Alternative Medicine, vol. 2013, Article ID 147362, 14 pages, 2013.

[37] Y. Lv and L. Fu, “The potential mechanism for Hydroxysafflor yellow A attenuating blood-brain barrier dysfunction via tight junction signaling pathways excavated by an integrated serial affinity chromatography and shotgun proteomics analysis approach," Neurochemistry International, vol. 112, pp. 38-48, 2018.

[38] P.-P. He, F.-H. Fu, T. Wang, C.-K. Li, W.-Y. Xin, and X.M. Zhang, "Effect of cerebral ischemia/reperfusion injury on hydroxysafflor yellow a penetrating across the blood-brain barrier," Scientia Pharmaceutica, vol. 76, no. 4, pp. 713-723, 2008.

[39] L. Sun, L. Yang, and Y.-W. Xu, "Neuroprotection of hydroxysafflor yellow $\mathrm{A}$ in the transient focal ischemia: inhibition of protein oxidation/nitration, 12/15-lipoxygenase and blood-brain barrier disruption," Brain Research, vol. 1473, no. 9, pp. 227-235, 2012.

[40] L. Sun, L. Yang, Y. Fu et al., "Capacity of HSYA to inhibit nitrotyrosine formation induced by focal ischemic brain injury," Nitric Oxide: Biology and Chemistry, vol. 35, pp. 144-151, 2013.

[41] L. Fan, X. Dang, Z. Shi, C. Zhang, and K. Wang, "Hydroxysafflor Yellow A Protects PC12 Cells Against the Apoptosis Induced by Oxygen and Glucose Deprivation," Cellular and Molecular Neurobiology, vol. 31, no. 8, pp. 1187-1194, 2011.

[42] Y. Lv, Y. Qian, A. Ou-yang, and L. Fu, "Hydroxysafflor Yellow A attenuates neuron damage by suppressing the lipopolysaccharide-induced TLR4 pathway in activated microglial cells," Cellular and Molecular Neurobiology, vol. 36, no. 8, pp. 1241-1256, 2016.

[43] Q. Yang, Z.-F. Yang, S.-B. Liu et al., "Neuroprotective effects of hydroxysafflor yellow A against excitotoxic neuronal death partially through down-regulation of NR2B-containing NMDA receptors," Neurochemical Research, vol. 35, no. 9, pp. 1353-1360, 2010.

[44] X. Wang, Z. Ma, Z. Fu et al., "Hydroxysafflor yellow A protects neurons from excitotoxic death through inhibition of NMDARs," Asn Neurochemistry, vol. 8, no. 2, Article ID 1759091416642345, 2016.

[45] J. W. Tian, F. H. Fu, W. L. Jiang et al., "Protective effect of hydroxysafflor yellow A against rat cortex mitochondrial injuries induced by cerebral ischemia," Acta Pharmaceutica Sinica, vol. 39, no. 10, pp. 774-777, 2004.

[46] S. Ramagiri and R. Taliyan, "Neuroprotective effect of hydroxy safflor yellow A against cerebral ischemia-reperfusion injury in rats: Putative role of mPTP," Journal of Basic and Clinical Physiology and Pharmacology, vol. 27, no. 1, pp. 1-8, 2016.

[47] J. Tian, G. Li, Z. Liu, and F. Fu, "Hydroxysafflor yellow A inhibits rat brain mitochondrial permeability transition pores by a free radical scavenging action," Pharmacology, vol. 82, no. 2, pp. 121126, 2008.

[48] N. Zhang, M. Xing, Y. Wang et al., "Hydroxysafflor yellow A improves learning and memory in a rat model of vascular dementia by increasing VEGF and NR1 in the hippocampus," Neuroscience Bulletin, vol. 30, no. 3, pp. 417-424, 2014. 
[49] M. Xing, Q. Sun, Y. Wang, Y. Cheng, and N. Zhang, "Hydroxysafflor yellow A increases BDNF and NMDARs in the hippocampus in a vascular dementia rat model," Brain Research, vol. 1642, pp. 419-425, 2016.

[50] Y.-Q. Lu, Y. Luo, Z.-F. He et al., "Hydroxysafflor yellow a ameliorates homocysteine-induced alzheimer-like pathologic dysfunction and memory/synaptic disorder," Rejuvenation Research, vol. 16, no. 6, pp. 446-452, 2013.

[51] Z. Zhang, Z. Wu, X. Zhu, X. Hui, J. Pan, and Y. Xu, "Hydroxysafflor yellow A inhibits neuroinflammation mediated by A $\beta_{1-42}$ in BV-2 cells," Neuroscience Letters, vol. 562, pp. 39-44, 2014.

[52] Z.-H. Zhang, L.-J. Yu, X.-C. Hui et al., "Hydroxy-safflor yellow A attenuates $\mathrm{A} \beta_{1-42}$-induced inflammation by modulating the JAK2/STAT3/NF- $\kappa$ B pathway," Brain Research, vol. 1563, pp. $72-$ 80, 2014.

[53] S.-Z. Kong, Y.-F. Xian, S.-P. Ip et al., "Protective effects of hydroxysafflor yellow a on $\beta$-amyloid-induced neurotoxicity in PC12 cells," Neurochemical Research, vol. 38, no. 5, pp. 951-960, 2013.

[54] Y. Wang, C. Zhang, W. Peng et al., "Hydroxysafflor yellow A exerts antioxidant effects in a rat model of traumatic brain injury," Molecular Medicine Reports, vol. 14, no. 4, pp. 36903696, 2016.

[55] X.-D. Bie, J. Han, and H.-B. Dai, "Effects of hydroxysafflor yellow A on the experimental traumatic brain injury in rats," Journal of Asian Natural Products Research, vol. 12, no. 3, pp. 239-247, 2010.

[56] Y. Pan, D.-Y. Zheng, S.-M. Liu et al., "Hydroxysafflor yellow A attenuates lymphostatic encephalopathy-induced brain injury in rats," Phytotherapy Research, vol. 26, no. 10, pp. 1500-1506, 2012.

[57] J. P. Pei, L. H. Fan, K. Nan et al., "HSYA alleviates secondary neuronal death through attenuating oxidative stress, inflammatory response, and neural apoptosis in SD rat spinal cord compression injury," Journal of Neuroinflammation, vol. 14, no. 1, Article ID 97, 2017.

[58] B. Han, J. Hu, J. Shen, Y. Gao, Y. Lu, and T. Wang, "Neuroprotective effect of hydroxysafflor yellow A on 6-hydroxydopamineinduced Parkinson's disease in rats," European Journal of Pharmacology, vol. 714, no. 1-3, pp. 83-88, 2013.

[59] T. Wang, L. Wang, C. Li et al., "Hydroxysafflor Yellow A Improves Motor Dysfunction in the Rotenone-Induced Mice Model of Parkinson's Disease," Neurochemical Research, vol. 42, no. 5, pp. 1325-1332, 2017.

[60] T. Wang, S.-J. Duan, S.-Y. Wang et al., "Coadministration of hydroxysafflor yellow A with levodopa attenuates the dyskinesia," Physiology \& Behavior, vol. 147, pp. 193-197, 2015.

[61] X. Q. Song, L. N. Su, H. P. Wei et al., "Protective effects of hydroxysafflor yellow A against oxidative damage of $\beta$ mercaptoethanol during neural differentiation of mesenchymal stem cells," Chinese Herbal Medicines, vol. 9, no. 3, pp. 282-288, 2017.

[62] Y. He, Q. Liu, Y. Li et al., "Protective effects of hydroxysafflor yellow A (HSYA) on alcohol-induced liver injury in rats," Journal of Physiology and Biochemistry, vol. 71, no. 1, pp. 69-78, 2015.

[63] Y.-B. Zhang, H.-Y. Dong, X.-M. Zhao et al., "Hydroxysafflor yellow A attenuates carbon tetrachloride-induced hepatic fibrosis in rats by inhibiting Erk5 signaling," American Journal of Chinese Medicine, vol. 40, no. 3, pp. 481-494, 2012.

[64] Y. Li, Y. Shi, Y. Sun et al., "Restorative effects of hydroxysafflor yellow A on hepatic function in an experimental regression model of hepatic fibrosis induced by carbon tetrachloride," Molecular Medicine Reports, vol. 15, no. 1, pp. 47-56, 2017.

[65] C. Y. Wang, Q. Liu, Q. X. Huang et al., "Activation of PPAR $\gamma$ is required for hydroxysafflor yellow A of Carthamus tinctorius to attenuate hepatic fibrosis induced by oxidative stress," Phytomedicine, vol. 20, no. 7, pp. 592-599, 2013.

[66] Q. Liu, C. Y. Wang, Z. Liu et al., "Hydroxysafflor yellow A suppresses liver fibrosis induced by carbon tetrachloride with high-fat diet by regulating PPAR- $\gamma /$ p38 MAPK signaling," Pharmaceutical Biology, vol. 52, no. 9, pp. 1085-1093, 2014.

[67] J. Trebicka, M. Hennenberg, M. Odenthal et al., "Atorvastatin attenuates hepatic fibrosis in rats after bile duct ligation via decreased turnover of hepatic stellate cells," Journal of Hepatology, vol. 53, no. 4, pp. 702-712, 2010.

[68] H. Dong, Y. Liu, Y. Zou et al., "Alteration of the ERK5 pathway by hydroxysafflor yellow A blocks expression of MEF2C in activated hepatic stellate cells in vitro: Potential treatment for hepatic fibrogenesis," Pharmaceutical Biology, vol. 52, no. 4, pp. 435-443, 2014.

[69] C.-C. Li, C.-Z. Yang, X.-M. Li et al., "Hydroxysafflor yellow A induces apoptosis in activated hepatic stellate cells through ERK1/2 pathway in vitro," European Journal of Pharmaceutical Sciences, vol. 46, no. 5, pp. 397-404, 2012.

[70] S. Jiang, Z. Shi, C. Li, C. Ma, X. Bai, and C. Wang, "Hydroxysafflor yellow A attenuates ischemia/reperfusion-induced liver injury by suppressing macrophage activation," International Journal of Clinical and Experimental Pathology, vol. 7, no. 5, pp. 2595-2608, 2014.

[71] H. M> Piao, Q. F. Xue, J. Z. Jiang et al., "Hydroxysafflor yellow $A$ attenuates allergic airway inflammation by suppressing the activity of nuclear factor-kappa B in ovalbumin-induced asthmatic mice," International Journal of Clinical and Experimental Pathology, vol. 9, no. 11, pp. 21595-21604, 2016.

[72] Y. Wang, C. Xue, F. Dong et al., "Hydroxysafflor yellow A attenuates small airway remodeling in a rat model of chronic obstructive pulmonary disease," Biological \& Pharmaceutical Bulletin, vol. 37, no. 10, pp. 1591-1598, 2014.

[73] X. F. Wang, M. Jin, J. Tong et al., "Protective effect of hydroxysafflor yellow A against acute lung injury induced by oleic acid and lipopolysaccharide in rats," Acta Pharmaceutica Sinica, vol. 45, no. 7, pp. 940-944, 2010.

[74] C. Wang, Q. Huang, C. Wang et al., "Hydroxysafflor yellow A suppress oleic acid-induced acute lung injury via protein kinase A," Toxicology and Applied Pharmacology, vol. 272, no. 3, pp. 895-904, 2013.

[75] C. Wang, C. Wang, C. Ma et al., "Hydroxysafflor yellow A of Carthamus tinctorius attenuates lung injury of aged rats exposed to gasoline engine exhaust by down-regulating platelet activation," Phytomedicine, vol. 21, no. 3, pp. 199-206, 2014.

[76] C.-Y. Sun, C.-Q. Pei, B.-X. Zang, L. Wang, and M. Jin, “The ability of hydroxysafflor yellow A to attenuate lipopolysaccharideinduced pulmonary inflammatory injury in mice," Phytotherapy Research, vol. 24, no. 12, pp. 1788-1795, 2010.

[77] Y.-L. Liu, Y.-J. Liu, Y. Liu et al., "Hydroxysafflor yellow A ameliorates lipopolysaccharide-induced acute lung injury in mice via modulating toll-like receptor 4 signaling pathways," International Immunopharmacology, vol. 23, no. 2, pp. 649-657, 2014.

[78] Y. Zhang, L. Song, R. Pan, J. Gao, B.-X. Zang, and M. Jin, "Hydroxysafflor yellow A alleviates lipopolysaccharide-induced acute respiratory distress syndrome in mice," Biological \& Pharmaceutical Bulletin, vol. 40, no. 2, pp. 135-144, 2017. 
[79] Y. Wu, L. Wang, M. Jin, and B.-X. Zang, "Hydroxysafflor yellow A alleviates early inflammatory response of bleomycin-induced mice lung injury," Biological \& Pharmaceutical Bulletin, vol. 35, no. 4, pp. 515-522, 2012.

[80] M. Jin, L. Wang, Y. Wu, B.-X. Zang, and L. Tan, "Protective effect of hydroxysafflor yellow A on bleomycin- induced pulmonary inflammation and fibrosis in rats," Chinese Journal of Integrative Medicine, vol. 24, no. 1, pp. 32-39, 2018.

[81] M. Jin, Y. Wu, L. Wang, B. Zang, and L. Tan, "Hydroxysafflor Yellow A Attenuates Bleomycin-induced Pulmonary Fibrosis in Mice," Phytotherapy Research, vol. 30, no. 4, pp. 577-587, 2016.

[82] L. J. Song, Y. Zhu, M. Jin et al., "Hydroxysafflor yellow a inhibits lipopolysaccharide-induced inflammatory signal transduction in A549 cells," Fitoterapia, vol. 84, no. 1, pp. 107-114, 2013.

[83] R. Pan, Y. Zhang, B. Zang, L. Tan, and M. Jin, "Hydroxysafflor yellow A inhibits TGF- $\beta 1$-induced activation of human fetal lung fibroblasts in vitro," Journal of Pharmacy and Pharmacology, vol. 68, no. 10, pp. 1320-1330, 2016.

[84] R. Y. Pan, Y. Zhang, M. Zheng, B. Zang, and M. Jin, "Hydroxysafflor yellow A suppresses MRC-5 cell activation induced by TGF- $\beta 1$ by blocking TGF- $\beta 1$ binding to T $\beta$ RII," Frontiers in Pharmacology, vol. 8, Article ID 264, 2017.

[85] Y. Li, Y. Wu, Y. Guan, Z. Wang, and L. Zhang, "Hydroxysafflor yellow A induces apoptosis in MCF-7 cells by blocking $\mathrm{NF} \kappa \mathrm{B} / \mathrm{p} 65$ pathway and disrupting mitochondrial transmembrane potential," RSC Advances, vol. 4, no. 88, pp. 47576-47586, 2014.

[86] L. Liu, N. Si, Y. Ma et al., "Hydroxysafflor-yellow a induces human gastric carcinoma BGC-823 cell apoptosis by activating peroxisome proliferator-activated receptor gamma (PPAR $\gamma)$," Medical Science Monitor, vol. 24, pp. 803-811, 2018.

[87] J. Wang, J. Wang, X. Wang et al., "Molecular mechanism of inhibition of the abnormal proliferation of human umbilical vein endothelial cells by hydroxysafflor-yellow A," Pharmaceutical Biology, vol. 54, no. 7-9, pp. 1800-1807, 2016.

[88] S.-Y. Xi, Q. Zhang, C.-Y. Liu, H. Xie, L.-F. Yue, and X.-M. Gao, "Effects of hydroxy safflower Yellow-A on tumor capillary angiogenesis in transplanted human gastric adenocarcinoma BGC-823 tumors in nude mice," Journal of Traditional Chinese Medicine, vol. 32, no. 2, pp. 243-248, 2012.

[89] F. Yang, J. Li, J. Zhu, D. Wang, S. Chen, and X. Bai, "Hydroxysafflor yellow A inhibits angiogenesis of hepatocellular carcinoma via blocking ERK/MAPK and NF- $\kappa$ B signaling pathway in $\mathrm{H} 22$ tumor-bearing mice," European Journal of Pharmacology, vol. 754, pp. 105-114, 2015.

[90] L. Ma, L. Liu, Y. C. Ma et al., "The role of E-Cadherin/ $\beta$-Catenin in Hydroxysafflor Yellow A inhibiting adhesion, invasion, migration and lung metastasis of hepatoma cells," Biological \& Pharmaceutical Bulletin, vol. 40, no. 10, pp. 1706-1715, 2017.

[91] X. Zhang, D. Shen, Z.-R. Lü et al., "Effects of hydroxysafflor yellow A on ALDH1: Inhibition kinetics and molecular dynamics simulation," Process Biochemistry, vol. 49, no. 10, pp. 1664-1672, 2014.

[92] R. S. Ahima and J. S. Flier, "Adipose tissue as an endocrine organ," Trends in Endocrinology \& Metabolism, vol. 11, no. 8, pp. 327-332, 2000.

[93] H.-J. Zhu, L.-J. Wang, X.-Q. Wang et al., "Hormone-sensitive lipase is involved in the action of hydroxysafflor yellow A (HYSA) inhibiting adipogenesis of 3T3-L1cells," Fitoterapia, vol. 93, pp. 182-188, 2014.

[94] Y. Xu, J. Lee, Y.-D. Park, J.-M. Yang, J. Zheng, and Q. Zhang, "Molecular dynamics simulation integrating the inhibition kinetics of hydroxysafflor yellow A on $\alpha$-glucosidase," Journal of Biomolecular Structure and Dynamics, vol. 36 , no. 4, pp. 830840, 2018.

[95] N. Ahmed, "Advanced glycation endproducts-role in pathology of diabetic complications," Diabetes Research and Clinical Practice, vol. 67, no. 1, pp. 3-21, 2005.

[96] Z. Ni, Z. Zhuge, W. Li, H. Xu, Z. Zhang, and H. Dai, "Inhibitory effects of hydroxysafflor yellow A on the formation of advanced glycation end products in vitro," Biological \& Pharmaceutical Bulletin, vol. 35, no. 11, pp. 2050-2053, 2012.

[97] W. Li, J. Liu, P. He et al., "Hydroxysafflor yellow A protects methylglyoxal-induced injury in the cultured human brain microvascular endothelial cells," Neuroscience Letters, vol. 549, pp. 146-150, 2013.

[98] T. Chen, N. Chen, N. Pang et al., "Hydroxysafflor yellow A promotes angiogenesis via the angiopoietin 1/ Tie-2 signaling pathway," Journal of Vascular Research, vol. 53, no. 5-6, pp. 245254, 2016.

[99] F. Ye, J. Wang, W. Meng et al., "Proteomic investigation of effects of hydroxysafflor yellow A in oxidized low-density lipoproteininduced endothelial injury," Scientific Reports, vol. 7, no. 1, Article ID 17981, 2017.

[100] M. Jin, C.-Y. Sun, and B.-X. Zang, "Hydroxysafflor yellow A attenuate lipopolysaccharide-induced endothelium inflammatory injury," Chinese Journal of Integrative Medicine, vol. 22, no. 1, pp. 36-41, 2016.

[101] D. B. Ji, M. C. Zhu, B. Zhu et al., "Hydroxysafflor yellow A enhances survival of vascular endothelial cells under hypoxia via upregulation of the HIF- $1 \alpha$-VEGF pathway and regulation of Bcl-2/Bax," Journal of Cardiovascular Pharmacology, vol. 52, no. 2, pp. 191-202, 2008.

[102] D. B. Ji, L. Y. Zhang, C. L. Li, J. Ye, and H. B. Zhu, "Effect of Hydroxysafflor yellow A on human umbilical vein endothelial cells under hypoxia," Vascular Pharmacology, vol. 50, no. 3-4, pp. 137-145, 2009.

[103] X. L. Xu, Y. H. Guo, J. X. Zhao et al., "Hydroxysafflor yellow A inhibits LPS-induced NLRP3 inflammasome activation via binding to xanthine oxidase in mouse RAW264.7 macrophages," Mediators of Inflammation, vol. 2016, Article ID 8172706, 2016.

[104] Yueqing Jiang, Jiayuan Kou, Xiaobo Han et al., "ROS-dependent activation of autophagy through the PI3K/Akt/mTOR pathway is induced by hydroxysafflor yellow A-sonodynamic therapy in THP-1 macrophages," Oxidative Medicine and Cellular Longevity, vol. 2017, Article ID 8519169, 16 pages, 2017.

[105] W. Wu, M. Jin, J. Tong et al., "Inhibitory effect of hydroxysafflor yellow A against PMN activation induced by LPS," Acta Pharmaceutica Sinica, vol. 46, no. 2, pp. 153-157, 2011.

[106] B. Cheng, Y. Gao, J. Lian et al., "Hydroxysafflor yellow A inhibits IL- $1 \beta$-induced release of IL-6, IL-8, and MMP-1 via suppression of ERK, NF- $\kappa$ B and AP-1 signaling in SW982 human synovial cells," Food \& Function, vol. 7, no. 11, pp. 4516-4522, 2016.

[107] F. Dong, C. Xue, Y. Wang et al., "Hydroxysafflor yellow A attenuates the expression of inflammatory cytokines in acute soft tissue injury," Scientific Reports, vol. 7, no. 1, Article ID 40584, 2017.

[108] J. Wang, P. Wang, S. Q. Gui et al., "Hydroxysafflor yellow A attenuates the apoptosis of peripheral blood CD4+T lymphocytes in a murine model of sepsis," Frontiers in Pharmacology, vol. 8, Article ID 613, 2017. 
[109] N. Hu, J. Duan, H. Li et al., "Hydroxysafflor Yellow A Ameliorates Renal Fibrosis by Suppressing TGF- $\beta 1$-Induced Epithelialto-Mesenchymal Transition," PLoS ONE, vol. 11, no. 4, Article ID e0153409, 2016.

[110] S.-Z. Kong, X.-G. Shi, X.-X. Feng et al., "Inhibitory effect of hydroxysafflor yellow A on mouse skin photoaging induced by ultraviolet irradiation," Rejuvenation Research, vol. 16, no. 5, pp. 404-413, 2013.

[111] S.-J. Yin, K.-Y. Liu, J. Lee et al., "Effect of hydroxysafflor yellow A on tyrosinase: integration of inhibition kinetics with computational simulation," Process Biochemistry, vol. 50, no. 12, pp. 2112-2120, 2015.

[112] Z. Liu, C. Li, M. Li, D. Li, and K. Liu, "The subchronic toxicity of hydroxysafflor yellow A of 90 days repeatedly intraperitoneal injections in rats," Toxicology, vol. 203, no. 1-3, pp. 139-143, 2004. 


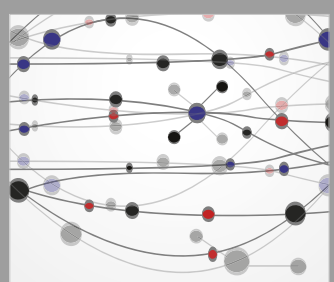

The Scientific World Journal
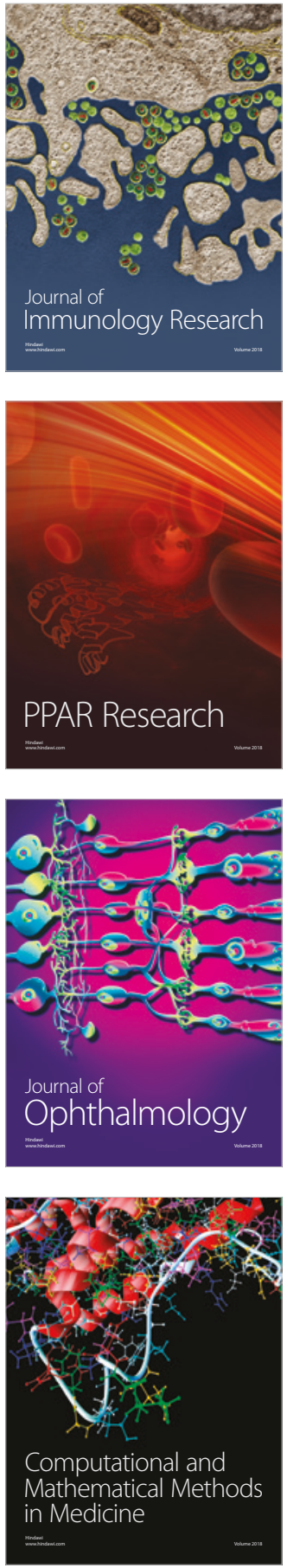

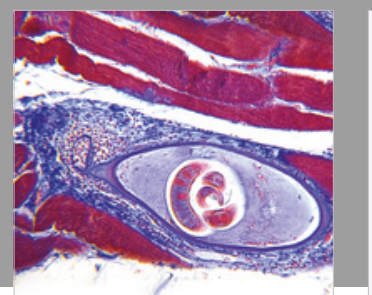

Gastroenterology Research and Practice

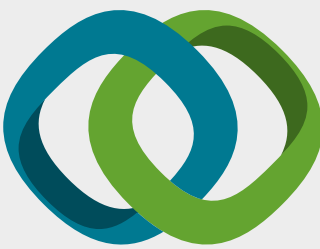

\section{Hindawi}

Submit your manuscripts at

www.hindawi.com
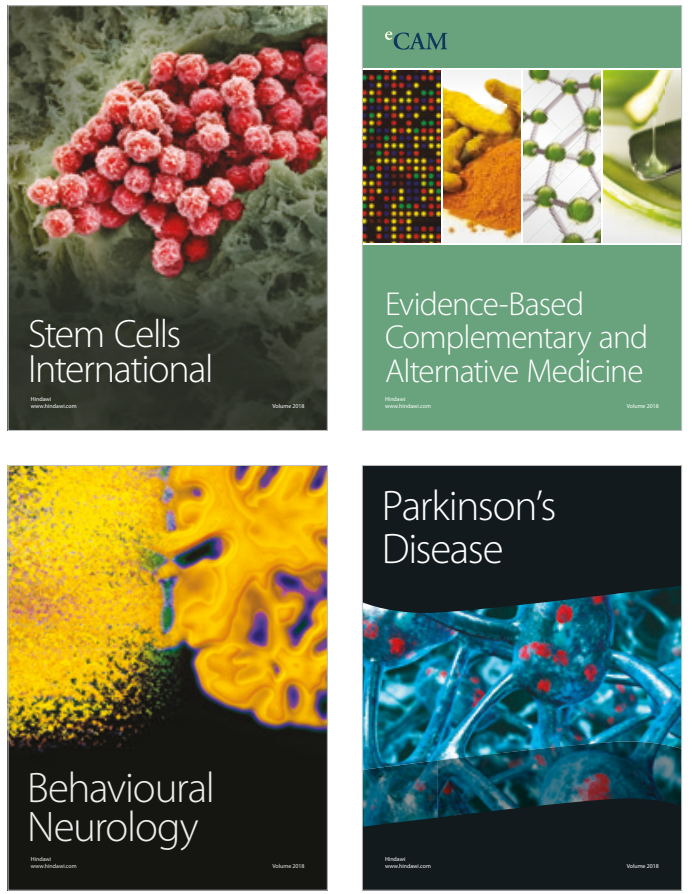

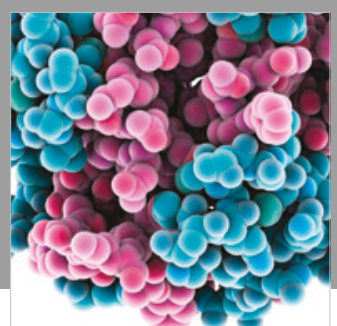

ournal of

Diabetes Research

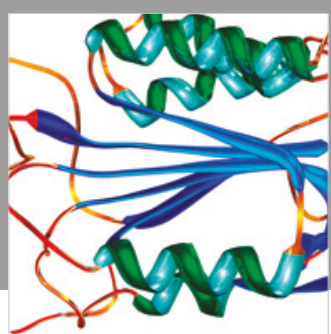

Disease Markers
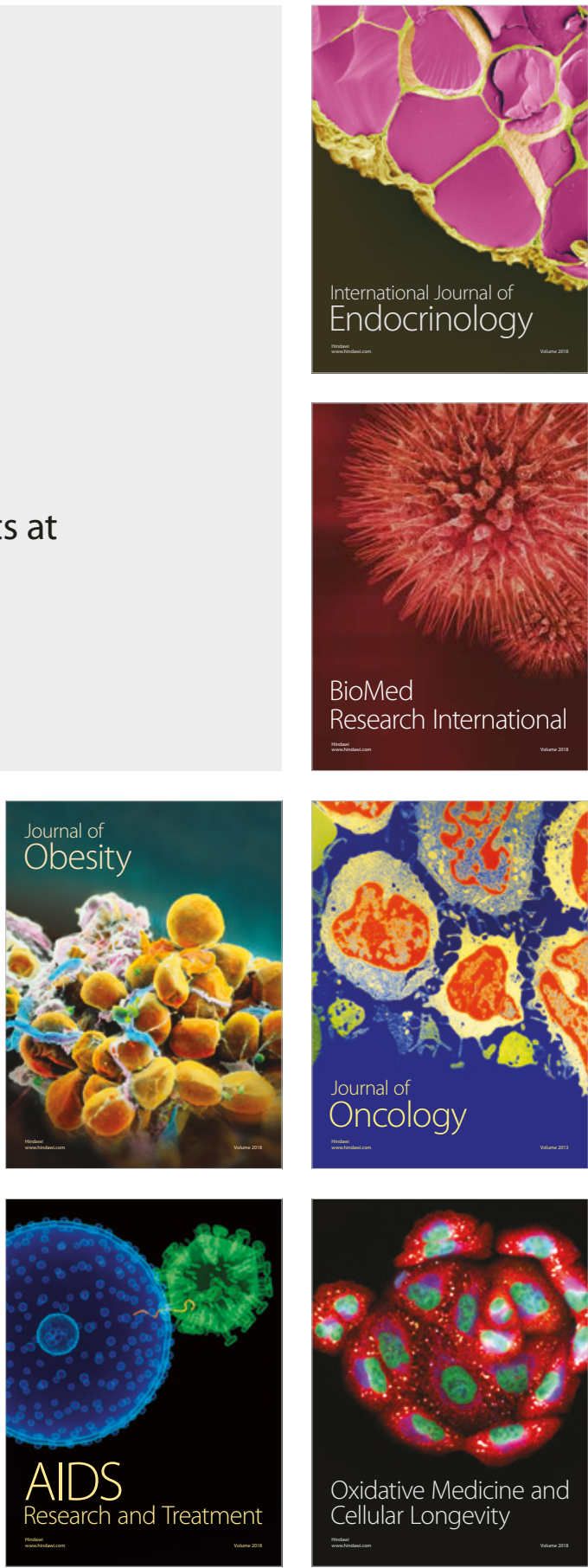